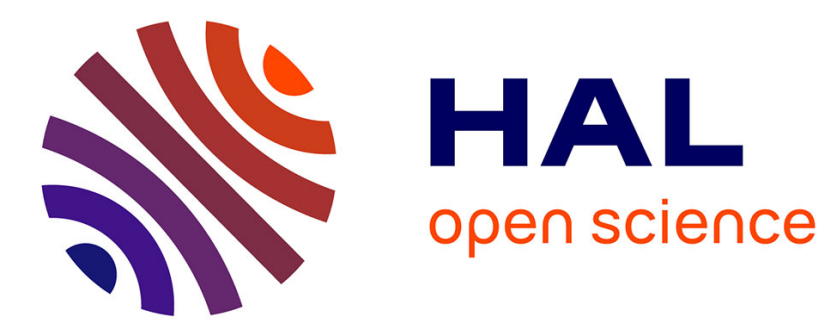

\title{
Expérimenter l'(extra)ordinaire : retour sur la ville nouvelle en Chine continentale
}

\author{
Carine Henriot
}

\section{To cite this version:}

Carine Henriot. Expérimenter l'(extra)ordinaire : retour sur la ville nouvelle en Chine continentale. Les Cahiers d'Outre-Mer. Revue de géographie de Bordeaux, 2020, LXXIII (282), pp.115-152. 10.4000/com.12173 . hal-03369774

\section{HAL Id: hal-03369774 https://hal.science/hal-03369774}

Submitted on 7 Oct 2021

HAL is a multi-disciplinary open access archive for the deposit and dissemination of scientific research documents, whether they are published or not. The documents may come from teaching and research institutions in France or abroad, or from public or private research centers.
L'archive ouverte pluridisciplinaire HAL, est destinée au dépôt et à la diffusion de documents scientifiques de niveau recherche, publiés ou non, émanant des établissements d'enseignement et de recherche français ou étrangers, des laboratoires publics ou privés. 


\title{
Les Cahiers d'outre-Mer, dossier :
}

- " Des villes nouvelles et des Suds : trajectoires à longs termes. Modèles, gouvernances, territorialités "

$\mathbf{u}_{\mathbf{B}}$
CAIRN

MATIÈRES À RÉFLEXION

\section{EXPÉRIMENTER L'(EXTRA)ORDINAIRE : RETOUR SUR LA VILLE NOUVELLE EN CHINE CONTINENTALE}

\author{
Carine Henriot
}

Presses universitaires de Bordeaux | «Les Cahiers d'Outre-Mer »

$2020 / 2 n^{\circ} 282$ | pages 115 à 152

ISSN 0373-5834

ISBN 9791030007008

Article disponible en ligne à l'adresse :

https://www.cairn.info/revue-les-cahiers-d-outre-mer-2020-2-page-115.htm

Distribution électronique Cairn.info pour Presses universitaires de Bordeaux.

(c) Presses universitaires de Bordeaux. Tous droits réservés pour tous pays.

\begin{abstract}
La reproduction ou représentation de cet article, notamment par photocopie, n'est autorisée que dans les limites des conditions générales d'utilisation du site ou, le cas échéant, des conditions générales de la licence souscrite par votre établissement. Toute autre reproduction ou représentation, en tout ou partie, sous quelque forme et de quelque manière que ce soit, est interdite sauf accord préalable et écrit de l'éditeur, en dehors des cas prévus par la législation en vigueur en France. Il est précisé que son stockage dans une base de données est également interdit.
\end{abstract}

Auteur: Carine HENRIOT, Maître de conférences en aménagement de l'espace et urbanisme, Alliance Sorbonne université - Université de technologie de Compiègne, EA 7284 AVENUES | carine.henriot@utc.fr.

Biographie : Carine Henriot est maître de conférences en aménagement de l'espace et en urbanisme, au sein du département de Génie urbain de Sorbonne universités, Université de technologie de Compiègne, chercheur à l'EA 7284 Avenues. Ses travaux portent sur la production de la ville chinoise et française, à travers la circulation de modèles urbains, les politiques d'urbanisme et les pratiques de la planification territoriale, avec une focalisation sur le numérique et l'énergie.

| carine.henriot@utc.fr. 
Titre de l'article : Expérimenter l'(extra)ordinaire : retour sur la ville nouvelle en Chine continentale

Mots-clés (x5) : ville nouvelle, politique d'urbanisme, modèle urbain, circulation de modèle, Chine

Résumé (x8 lignes) : En Chine continentale, comme ailleurs en Asie, la ville nouvelle constitue une politique d'urbanisme appartenant autant à l'histoire de l'urbanisme qu'à l'actualité de la planification urbaine. Cette contribution propose une synthèse de nos arpentages des villes nouvelles chinoises, que nous alimentons de nos lectures sur ces urbanités hors norme, et en creux de la production urbaine (extra)ordinaire chinoise. Nous montrerons que la figure de la ville nouvelle circule, influence et renouvelle la production urbaine et l'urbanisme chinois, depuis le milieu du XXe siècle jusqu'à nos jours, en dessinant une trajectoire chinoise de la ville nouvelle, à la fois caractéristique de la trajectoire urbaine chinoise et intégrée à la mondialisation de la ville nouvelle.

Title : Experiencing the (extra)ordinary: back to the new town in mainland China

Key words: new town/ new city, urban planning policy, urban model, model circulation, China

Abstract: In mainland China, as elsewhere in Asia, the new town/city constitutes an urban planning policy that is as much a part of the history of urban planning as it is of current urban planning. This contribution proposes a synthesis of our fieldwork on Chinese new cities, which we feed from our reading of these unusual urbanities, and in the depths of the (extra)ordinary Chinese urban production. We will show that the figure of the new city circulates, influences and renews Chinese urban production and urban planning, from the middle of the 20th century to the present day, by drawing a Chinese trajectory of the new city, both characteristic of the Chinese urban trajectory and integrated into the globalization of the new city.

Plan :

I. De quelles villes nouvelles chinoises parle-t-on ?

I.1. Les villes nouvelles de desserrement métropolitain

I.2. La (dé)construction d'un corpus cohérent

I.3. Repartir de l'expérience du terrain : In situ, qu'est-ce qui « fait ville nouvelle » ?

1.4. Notre corpus idéal des cités nouvelles chinoises

II. Une première mondialisation de la ville nouvelle

II.1. Les premières expériences de desserrement résidentiel

II. 2. Les villes satellites de desserrement industriel

II. 3. Les villes nouvelles de desserrement métropolitain

III. Vers une seconde mondialisation de la ville nouvelle: des inflexions thématiques pour une urbanisation qualitative?

III.1. Les " nouveaux bourgs ", entre nouvel urbanisme et architecture néo-traditionnelle

III.2. Les villes nouvelles durables, un tournant environnemental limité

III.3. Des villes nouvelles intelligentes pour renouveler la production urbaine ?

Conclusion : Vers une trajectoire de la ville nouvelle chinoise de desserrement

\section{Introduction:}


La ville nouvelle en tant qu'outil d'aménagement et objet scientifique pluridisciplinaire a connu un engouement certain en France dans les années 1960-1970, avant de s'éclipser progressivement dans les années 1980. Depuis les années 2000, la question de la "ville nouvelle " est réinvestie par I'histoire urbaine contemporaine (Vadelorge 2005, 2014) et renouvelée par "les circulations internationales de modèles et d'expériences " (Vadelorge, 2017a, 2017b, 2017c ; Orillard 2017), pour désormais s'ouvrir sur les Suds (Ballout, 2019, Ballout, 2020).

En Chine continentale, comme ailleurs en Asie, la ville nouvelle constitue une politique d'urbanisme appartenant autant à l'histoire de l'urbanisme qu'à l'actualité de la planification urbaine. Les chercheurs chinois rendent en effet compte dès la fin des années 1950 de projets de planification et de construction de "villes satellites " pour décongestionner les principales villes chinoises (Funk, 1996: 338). Les Universités Tsinghua à Pékin et Tongji à Shanghai sont les seules universités chinoises à dispenser l'enseignement de l'architecture et de l'urbanisme durant la Révolution culturelle (Hoa, 1981 : 63-65). En raison de l'isolement scientifique des trente premières années du régime communiste, la production scientifique mentionne, tardivement, l'existence de villes satellites chinoises, en langue anglaise (Thompson, 1974: 152; Murphey, 1976: 324), comme française (Hoa, 1981). Par exemple, les premières publications scientifiques dédiées aux "villes satellites" de la municipalité de Shanghai sont publiées, par des géographes urbains et des urbanistes, en langue anglaise, dans les années 1980-1990 (Fung, 1981a, 1981b ; Kirkby, 1987 ; Ning et Yan, 1995 ; Funk, 1996), tandis que la première étude dédiée en langue française n'est rédigée qu'en 1991 (Li, 1991). Dans les années 2000, les architectes et urbanistes, chinois et internationaux, multiplient désormais les publications sur les "villes nouvelles " planifiées dans toute la Chine, en se focalisant sur leur composition urbaine et/ou leur architecture empruntées à l'Occident (Dell et Speer, 2002 ; Cagnardi, 2003 ; Wang et Li, 2006 ; Wang, 2007 ; Den Hartog at al., 2010 ; Liu, 2011 ; Henriot et Minost, 2017), et sur les inflexions des schémas directeurs et le fonctionnalisme (Billard, 2005 ; Den Hartog at al., 2010 ; Yu et Luo, 2009 ; Henriot, 2013a, 2016, 2017a ; Liu, 2014, 2018). Au milieu des années 2010, les villes nouvelles construites dix à quinze ans plus tôt sont désormais peuplées et intégrées aux banlieues chinoises. Les géographes urbains s'intéressent de nouveau à l'objet "ville nouvelle ", aux côtés des urbanistes et des architectes, pour interroger ses formes urbaines (Shen et Wu, 2012), son peuplement (Henriot, 2013a : 271-338, 2016, 2017b ; Liu, 2018), la politique chinoise de villes nouvelles et ses différents types (Tan, 2010 ; Henriot, 2015 ; Liu et Xu, 2019), l'outil d'aménagement dédié à travers les sociétés de développement des villes nouvelles (Henriot, 2013b ; Li et Chiu, 2018 ; Shen et Wu, 2017, Zhuang et Ye, 2018), le modèle et la circulation de ses références urbaines (Henriot, 2015; Henriot et Minost, 2017; De Pieri et Vero, 2019 ; Fiandanese, 2019). Un travail de synthèse remarquable sur " The City after Chinese New Towns ", à l'initiative de la "China room " de Politecnico di Turino, (Bonino, Governa, Repellino, Sampieri, 2019), vient enrichir nos connaissances sur la ville nouvelle chinoise, à partir de trois villes nouvelles contemporaines (Tongzhou à Pékin, Zhaoqing dans le delta de la rivière des perles et Zhengdong à Zhengzhou). L'ouvrage collectif, très renseigné et richement illustré, est le fruit d'un travail nécessairement pluridisciplinaire (architectes, urbanistes, sociologue et ethnologue); il renouvelle notre lecture de la ville chinoise, de la production de ses espaces et de ses imaginaires.

En réponse à l'appel à contributions publié par Jean-Marie Ballout, dans Cahiers d'Outre-Mer, nous proposons, modestement, de positionner cette contribution sur les villes nouvelles chinoises, en articulant à la question de la ville nouvelle de desserrement métropolitain (Merlin, 1988, rééed. 1996 ; Merlin et Sudarskis, 1991) celle du modèle en urbanisme (Choay, 1988), pour rendre compte, sur le temps long du second vingtième siècle, de l'émergence de ces villes nouvelles, comme figure de proue du développement urbain chinois. En effet, si lorsque nous nous sommes rendus sur le terrain pour la première fois, il nous semblait important de questionner l'apport des villes nouvelles chinoises au mouvement des new towns, en Asie et dans le monde, il nous apparaît aujourd'hui moins intéressant de questionner cet apport que celui des villes nouvelles à la production urbaine chinoise. Nous pouvons en effet observer la production urbaine, son contexte en termes de 
processus d'urbanisation, ses mécanismes de planification/conception/construction, ses formes urbaines et ses aspirations sociales, depuis l'expérience de la ville nouvelle, laquelle nous renseigne, par un jeu de miroir, sur ce qui l'entoure, à savoir, sur la production urbaine ordinaire chinoise. La figure de la ville, nouvelle, en tant qu'objet de planification, dessine en Chine, sur le second vingtième siècle, une trajectoire, laquelle nous renseigne sur le contexte du développement urbain chinois, sur les politiques locales d'urbanisme et sur les aspirations des classes solvables, notamment en termes de logement. Nous montrerons que cette trajectoire de la ville nouvelle chinoise est à la fois caractéristique de la trajectoire urbaine chinoise et intégrée à la mondialisation de la ville nouvelle. Pour cela, nous proposons, dans un premier temps, de préciser d'où nous parlons, de quelles villes nouvelles chinoises il sera question dans cette contribution.

\section{De quelles villes nouvelles chinoises parle-t-on?}

Il existe une réelle difficulté à se saisir de ce que sont les villes nouvelles - a fortiori, en périphérie des grandes villes - dans les pays connaissant une urbanisation tellement rapide qu'extension urbaine, nouveaux quartiers résidentiels, nouvelles zone industrielle, zone économique ou zone universitaire, et projets de ville nouvelle, à proprement parlé, se confondent. Dès lors, il nous semble primordial de préciser ce que nous entendons par "ville nouvelle de desserrement métropolitain ", d'identifier un corpus cohérent, puis de repartir du terrain, pour préciser: à quelles réalités matérielles (formes urbaines, réseaux urbains, notamment de transports), actorielles (décideurs, aménageurs/urbanistes/architectes, habitants), processuelles (urbanisation, planification, construction, peuplement et migrations résidentielles) renvoient ces villes nouvelles.

\section{I.1. Les villes nouvelles de desserrement métropolitain}

Le corpus retenu pour cette étude propose d'analyser des "villes nouvelles » chinoises, aménagées, par les gouvernements locaux, pour maîtriser la croissance des très grandes villes - par opposition aux villes nouvelles, créées ex-nihilo, dans le cadre de politiques nationales, pour fonder un nouveau centre de commandement politique et administratif ou créer un pôle de croissance régional, souvent une nouvelle ville industrielle (Merlin, 1988, rééed. 1996). Cette définition exclue les villes nouvelles industrielles construites sur les marches de la Chine dans une politique de conquête d'un vaste territoire ${ }^{1}$ et visant l'exploitation de ressources naturelles, suivant la logique des fronts pionniers soviétiques en Sibérie ou en Extrême-Orient russe (Kirkby, 1987 : 205). Selon la définition retenue, la ville nouvelle constitue un choix d'urbanisme raisonné, une extension urbaine périphérique volontaire et planifiée, dotée de fonctions urbaines complètes. Les villes nouvelles de desserrement constituent dès lors un outil opérationnel d'aménagement guidant le processus d'urbanisation vers une zone à urbaniser en priorité afin de limiter l'étalement urbain non contrôlé, de desserrer les activités et les populations, d'orienter la croissance urbaine vers des pôles identifiés, de rationaliser le zonage des fonctions urbaines. Ces villes nouvelles participent également à l'échelle de l'aire urbaine à l'organisation d'un dispositif polycentrique, où la ville-centre est relayée par des pôles secondaires de développement, dotés de compétences propres, favorisant l'intégration des nouvelles périphéries urbaines à la ville-centre (Henriot ; 2013b).

\section{I.2. La (dé)construction d'un corpus cohérent}

\footnotetext{
${ }^{1}$ « Dans les pays socialistes, plus encore que dans les pays occidentaux, la politique d'urbanisme est au service d'une politique d'aménagement du territoire, elle-même dictée par les nécessités de l'économie nationale. Dans ce contexte, les villes nouvelles, moins que des opérations d'urbanisme à situer dans le contexte de la croissance des grandes agglomérations, sont une forme d'habitat nécessaire pour permettre l'implantation d'une nouvelle industrie. " (Merlin, 1969, rééd. 1972 : 11-82).
} 
Le bornage temporel retenu (depuis la proclamation de la République populaire de Chine, en 1949) sort du champs d'analyse les apports des concessions étrangères ou quartiers extraterritoriaux britanniques, français, américains, allemands, italiens à l'architecture chinoise, au génie urbain et à l'urbanisme naissants entre les années 1860 et 1940. Nous considérons que ces quartiers constituent un corpus cohérent, à part entière, appartenant à l'histoire de l'urbanisme. Les premiers plans d'aménagement portés par les nationalistes dans les années 1930 et 1940 sont également hors champs d'analyse, car leurs plans sont rapidement abandonnés lors de la prise de pouvoir par les communistes.

Dans la période contemporaine, les villes nouvelles monofonctionnelles comme les cités universitaires (Li, Li et Wang, 2014 ; Ruoppilaa et Zhao, 2017) sont hors champs d'analyse. Les villes nouvelles monofonctionnelles relevant de politiques économiques, commerciales, ou douanières, d'échelle nationale, telles les zones économiques spéciales (special economic zone, SEZ) lancées à partir de 1979, les zones de développement économique et technologique (economic and technological development zone, ETDZ) lancées 1984, les zones de collaboration économiques frontalières (border economic collaboration area) lancées en 1992, les zones de développement industriel dédiées aux nouvelles technologies (hight-tech industrial development zone, HTIDZ) lancées en 1988, et les différentes zones douanières qui se succèdent à partir de $1990^{2}$, bien que reconnues par certains chercheurs comme des villes nouvelles à proprement parlé (Zhuang et $\mathrm{Ye}$, 2018 ; Liu et Xu, 2019 ; André, 2020a : 535) sont également hors champs d'analyse pour cette étude. Ces villes nouvelles constituent un corpus cohérent, à part entière, ne relevant pas des politiques locales d'urbanisme, mais de politiques nationales de développement économique, pilotées par le gouvernement central. De même, les villes nouvelles relevant des "nouveaux arrondissements " (xinqu ou new district), comme Pudong, le « nouvel arrondissement cristallisant la modernisation de Shanghai " (xiandaihua xinqu), lancé en 1992, sur $1210 \mathrm{~km}^{2}$, ou comme Binhai à Tianjin, lancé en 2006 , sur $644 \mathrm{~km}^{2}$, sont reconnus par les chercheurs chinois comme relevant d'une politique nationale de ville nouvelle particulière, dédiée aux réformes, et pilotée par la Commission pour les réformes et le développement (National Development and Reform Commission - NDRC), et constituent, une nouvelle fois, un corpus cohérent, à part entière en raison de leur périmètre souvent très vaste et des prérogatives dont ces territoires disposent (Liu et Xu, 2019 ; André, 2020 : 535). Outre ces villes nouvelles relevant d'une politique nationale, les villes durables et intelligentes planifiées dans le cadre d'une politique nationale, et non locale, et alimentées par des coopérations bilatérales, comme l'éco-cité sino-singapourienne de Tianjin (Curien, 2016 ; Li et al., 2018) ou l'écocité sino-française de Caidian à Wuhan (André, 2020a : 172-173; 2020b: document 7) sont hors champ. Elles ont disposé pour leur création d'une concentration de moyens et d'expertises les rapprochant d'expérimentations internationales - et seront convoquées en tant que tel. Elles ne peuvent être considérées comme des expériences locales d'aménagement, portées par les seuls arrondissements de banlieue.

Aussi, notre "corpus idéal " comprend des villes nouvelles faisant explicitement référence à un programme local de redéploiement métropolitain, visant la construction d'une entité urbaine, développée ex-nihilo ou adossée à un tissu urbain ancien, présentant une morphologie urbaine identifiable et des fonctions urbaines relativement diversifiées.

\section{I.3. Repartir de l’expérience du terrain : In situ, qu'est-ce qui « fait ville nouvelle » ?}

Lorsque nous nous sommes rendus sur le terrain pour la première fois, en 2007, à Pékin, à Shanghai et à Hong Kong, il nous semblait important de questionner l'apport des villes nouvelles chinoises au mouvement des new towns. Nourris par l'expérience des villes nouvelles franciliennes, nous

\footnotetext{
${ }^{2}$ Les zones douanières chinoises sont les : tax-protected zone (1990), export processing area (2000), bounded logistic park (2004), tax-protected port area (2005) et integrated tax-protected zone (2006) (Liu et Xu, 2019).
} 
imaginions les villes nouvelles chinoises comme de formidables laboratoires d'une nouvelle urbanité asiatique. Nous pensions explorer les banlieues chinoises et " reconnaître ", avec évidence, l'apport de ces villes nouvelles, que nous supposions cohérent. Nous avons rapidement compris qu'il ne saurait être question de comparer les villes nouvelles honkongaises et les villes nouvelles continentales. Ces villes ne participaient pas de la même production urbaine, parce qu'elles ne répondaient pas aux même objectifs, et n'avaient pas bénéficié de la même expertise nationale et internationale. Nous reviendrons sur ces expériences ultérieurement. De même, les villes nouvelles pékinoises et shanghaiennes ne pouvaient être que discutées et pas comparées à proprement parlé. Au fond, nous ne comprenions pas grand-chose aux formes urbaines juxtaposées qui s'étalaient en banlieue des villes que nous parcourions. En effet, la rapidité de construction, l'enchevêtrement des périmètres de planification et $l$ ' " assemblage " des nouveaux secteurs aménagés brouillaient notre lecture des périmètres d'intervention prioritaire que sont censés être les villes nouvelles. De même, au fur et à mesure que nous nous familiarisions avec la planification de développement urbain de Shanghai, nous avons réalisé, avec stupéfaction, que les périmètres d'intervention des villes nouvelles ne distinguent pas toujours dans leur schéma directeur les secteurs déjà urbanisés, en renouvellement urbain et en développement. II nous fallait repartir du terrain, pour comprendre ce que sont les villes nouvelles chinoises de desserrement métropolitain, pour comprendre ce qui « fait ville nouvelle » en Chine.

Je me souviens avoir commencé par approcher les villes nouvelles shanghaiennes en achetant toutes les cartes des arrondissements de banlieue de Shanghai, en listant tous les toponymes évoquant une ville nouvelle: "ville nouvelle» (xincheng), "petite ville nouvelle/bourg nouveau " (xinzhen), "nouveau village " (xincun). Puis, j'ai scrupuleusement visité chacune de ces "villes nouvelles", pour en apprécier les formes urbaines, l'insertion dans le tissu -notamment morphologique et fonctionnel- environnant, les fronts d'urbanisation. Cela m'a permis de me rendre compte que derrière ces toponymes se cachaient aussi bien de nouveaux secteurs aménagés, de manière prioritaire, dans le cadre de programmes variés de villes nouvelles, que des grandes opérations de promotion immobilières, cherchant, par cette terminologie, à assurer une valorisation certaine de leur projet. Certains de ces quartiers correspondaient même à des opérations de promotion immobilière bon marché, destinées à accueillir le desserrement résidentiel des populations déplacées du centre-ville lors d'opérations de renouvellement urbain.

J'ai souhaité pondérer cette expérience par d'autres références. Je me suis rendue dans plusieurs provinces chinoises appartenant aux ensembles régionaux du littoral, de l'intérieur, des périphéries. J'ai visité d'autres métropoles extrême-orientales, comme Séoul, et d'Asie du Sud-Est, comme Singapour, pour voir à quoi ressemble là-bas une ville nouvelle. Ces comparaisons et nouveaux angles d'approche, loin de me permettre d'identifier des caractéristiques communes aux villes nouvelles asiatiques, ou au contraire spécifiques à Shanghai, $\mathrm{m}^{\prime}$ ont permis de comprendre que les villes nouvelles chinoises, qu'elles soient considérées comme des expériences réussies ou au contraire peu abouties, sont l'expression d'une trame générique chinoise, de la matrice de la production urbaine chinoise, dont le paysage s'observe aussi bien à Shanghai qu'à Pékin, Harbin, Wuhan, Chongqing, Urumqi ou aux villes frontières de la Chine, comme à Suifenhe, Dandong, Ulan Ude ou Kashgar.

Dès lors, à l'heure où le terrain ne nous est plus accessible, pandémie mondiale de la Covid-19 oblige, cette contribution prend un résonnance particulière, pour s'inscrire comme une synthèse de nos arpentages des villes nouvelles chinoises, que nous alimentons de nos lectures sur ces urbanités hors norme, et en creux de la production urbaine (extra)ordinaire chinoise.

\section{I.4. Notre corpus idéal des cités nouvelles chinoises}


Ce corpus idéal agglomère aux cas d'études arpentés, des expériences découvertes dans la littérature et fantasmées, pour illustrer la trajectoire des cités nouvelles chinoises du second vingtième siècle. Les " nouveaux village " (xincun), cités ouvrières satellites, construits à partir des années 1950, comme Caoyang à Shanghai, les « villes satellites » (weixincheng), construites entre les années 1950 et les années 1990, comme Minhang, Baoshanwei ou Jinshanwei à Shanghai, comme Shijingshan ou Yanshan à Pékin, les "villes nouvelles" (xincheng), construites depuis les années 1980, comme Songjiang, Jiading ou Qingpu à Shanghai, comme Yichuang et Tongzhou à Pékin, Zhengdong à Zhengzhou, et les "nouveaux bourgs»(xinzhen), construits depuis le début des années 2000, comme Anting xinzhen, Pujiangzhen, Taiwushi xiaozhen ou Zhujiajiao xinzhen à Shanghai, les villes durables, comme l'éco-ville de Dongtan à Shanghai, et les villes intelligentes, comme Minhang à Shanghai et Hangzhou, nous semblent ainsi constituer un corpus idéal cohérent, pour cette étude.

Tableau 1 : Corpus des villes nouvelles chinoises de desserrement métropolitain

Type de ville nouvelle Exemple

- Cité ouvrière satellite (xincun) - Caoyang xincun (Shanghai)

- Nanyan fang, Shenghuo cun (Wuhan)

- Ville satellite (weixincheng) - Minhang, Baoshanwei, Jinshanwei (Shanghai) - Shijingshan, Yanshan (Pékin)

- Ville nouvelle (xincheng) - Songjiang, Jiading, Qingpu (Shanghai) - Daxing, Yizhuang, Tongzhou (Pékin) - Zhengdong (Zhengzhou)

- « Nouveau bourg » (xinzhen) - Anting xinzhen, Pujiangzhen, Taiwushi

- Ville durable (yongxu chengshi)

- Ville intelligente (zhihui chengshi) xiaozhen, Zhujiajiao xinzhen, (Shanghai) - Eco-ville de Dongtan (Shanghai)

- Minhang (Shanghai)

- Hangzhou

Certaines de ces villes nous sont bien connues, pour les avoir arpentées, à plusieurs reprises, voire en de multiples occasions, lors de terrains successifs entre 2007 et 2019. C'est le cas des villes nouvelles shanghaiennes, et au-delà de Chine de l'Est, lesquelles ont fait l'objet d'enquêtes de terrain approfondies, d'entretiens auprès des bureaux de planification, des architectes-urbanistes, mais également des populations pionnières venues s'installer dans ces nouveaux secteurs aménagés. D'autres n'ont été visitées qu'une seule fois ou bien ne nous sont connues que par nos lectures. Tongzhou, par exemple, ne nous est connu qu'à travers les travaux de Filippo Fiandanese (Fiandanese, 2019) et l'excellent ouvrage publié par les collègues de la China Room de Politecnico di Turino (Bonino et al., 2019). Wuhan, également, ne nous est connu qu'à travers nos discussions avec Georgina André et ses travaux nourrissants (André, 2016 ; 2020a ; 2020b). Toutes, enfin, ont été arpentées via BaiduDitu et son outil de visualisation 3D, répondant ainsi au besoin d'évasion exacerbé par le confinement de l'automne 2020.

Notre objectif n'est pas de réaliser une étude comparative de ces villes nouvelles, pour en dégager des caractéristiques communes, mais plutôt de rendre compte d'une mondialisation chinoise de la ville nouvelle, et au-delà asiatique.

\section{Une première mondialisation de la ville nouvelle}

En Asie, la mondialisation de la ville nouvelle appartient autant à l'histoire de l'urbanisme (Chaline, 1986 ; Philips et Yeh, 1987) qu'à la planification contemporaine (Keeton, 2011). En Asie, comme ailleurs, le second $X X^{e}$ siècle voit l'émergence d'une mondialisation de la ville nouvelle, laquelle nous renvoie à l'histoire et à l'influence des villes nouvelles britanniques (Asie orientale et Asie du Sud), 
soviétiques (Asie centrale), mais également françaises (plus ponctuellement, en Corée du Sud et au Vietnam). Des villes nouvelles sont planifiées, en contexte autoritaire, sur le territoire britannique de Hong Kong, au Japon, en Corée du Sud, dans la Cité-Etat de Singapour, ainsi qu'en Malaisie et aux Philippines (Philips et Yeh, 1987). L'influence des plans et des chartes des villes nouvelles londoniennes et secondairement franciliennes (Petit, 2002) est d'ailleurs explicitement mentionnée par les aménageurs et les chercheurs travaillant sur l'Asie orientale. Ces projets d'aménagement s'inscrivent tous dans un contexte de développement économique et urbain rapide, bien souvent accéléré par un autoritarisme politique facilitant la disponibilité et la mutabilité du foncier. Les villes satellites et villes nouvelles de la République populaire de Chine s'inscrivent-elles également dans cette mondialisation de la ville nouvelle?

\section{II.1. Les premières expériences de desserrement résidentiel}

Avant même les programmes de villes satellites puis de villes nouvelles, la construction de quartiers résidentiels satellites est planifiée en périphérie des principales grandes villes asiatiques pour palier l'important besoin en logements destinés au relogement de la population après les destructions de la Seconde Guerre mondiale.

Le Japon développe, par exemple, dès les années 1950, des satellites résidentiels, qualifiés de " villes dortoirs " (Nagamine, 1987). De même, en Corée du Sud, dans les années 1960, des satellites résidentiels sont construits autour de Séoul (Lee, 1987). La péninsule de Hong Kong, colonie britannique jusqu'à sa rétrocession à la République populaire de Chine en 1997, est directement influencée par la politique des New Towns londoniennes, puisque quatre années après le Greater London Plan, Hong Kong publie dès 1948 le Hong Kong Abercrombie Plan, qui a bénéficié de l'expertise des mêmes aménageurs britanniques. Ce schéma promeut le renouveau du centre-ville de l'île de Hong Kong et des quartiers populaires de Kowloon, le développement de villes nouvelles visant un desserrement résidentiel (Pun, 1987; Fouchier, 1993) destiné à accueillir l'arrivée de réfugiés, notamment nationalistes, en provenance de Chine continentale (Chow, 1991). En raison de la surreprésentation de la fonction résidentielle et du manque d'équipements, ces villes nouvelles s'apparentent plutôt, dans les années 1970, à des villes satellites (Yeh, 1987 ; Fouchier 1993).

En Chine, les premières expériences de desserrement adossent des cités ouvrières à de nouveaux secteurs industriels, en très proche périphérie des principales villes. Cette forme d'extension urbaine apparaît, par exemple à Shanghai, sous le toponyme cun " village » ou xincun, " nouveau village » et voit les banlieues résidentielles s'étendre avec monotonie sous la forme de vastes quartiers constitués de blocs d'habitations répétitifs de $R+2+C$ à cinq étages (Cao, $1994: 270$; Ged, $2000: 35$; Bergère, 2002 : 398). Le premier village de la cité ouvrière de Caoyang xincun est ainsi construit à partir de 1951 (Photo 1 et 2).

[Photo 1, 2 : La cité ouvrière de Caoyang xincun, le premier village est construit à partir de 1951, à Shanghai. 19 août 2016, C. Henriot.] 


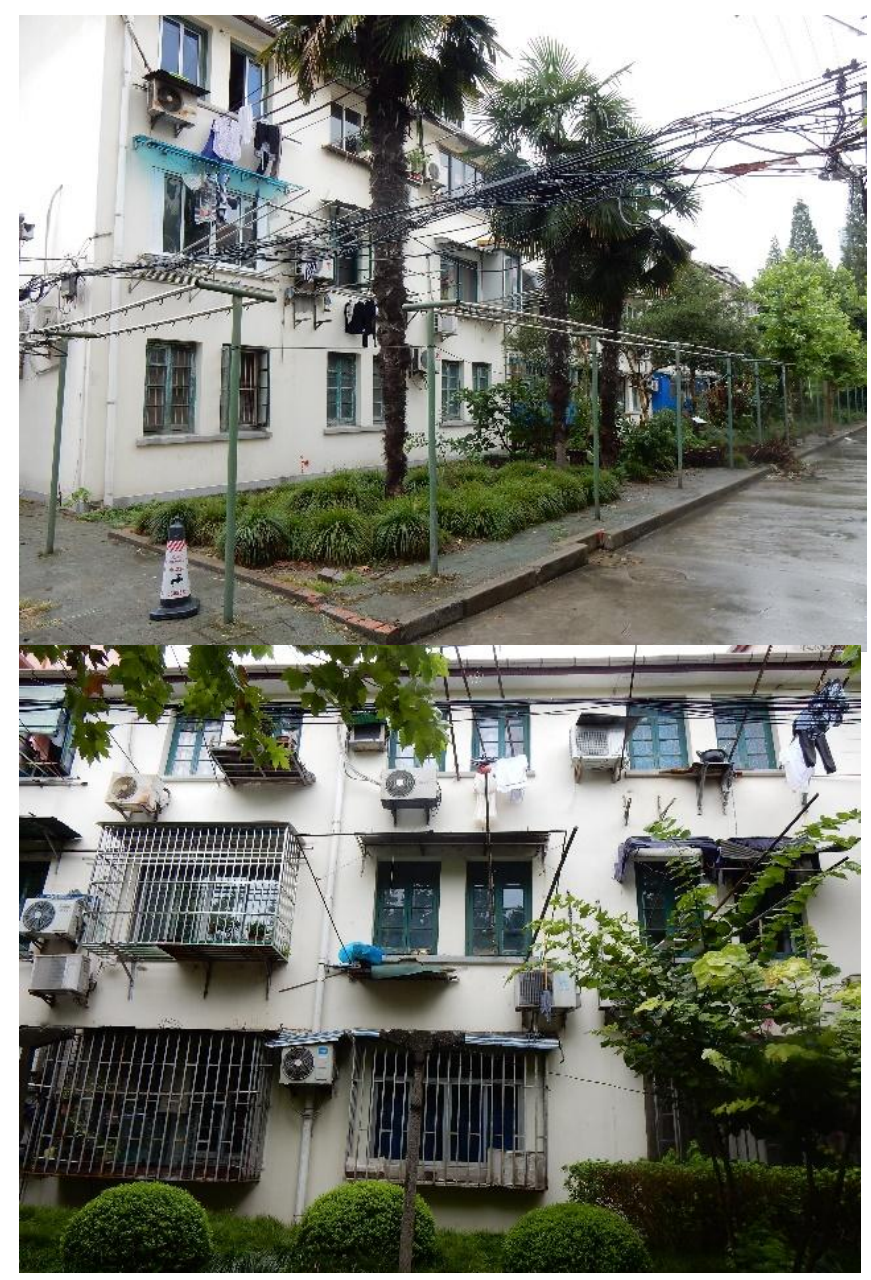

Autre exemple, à Wuhan, dès 1952, 4 nouveaux villages sont construits à Wuche (Andre, 2020a : 412). Au-delà, ce sont deux types de logements qui sont construits à destination des ouvriers : un type de logement permanent les hong fangzi, littéralement "maison rouge ", basés sur le modèle sino-soviétique d'organisation du bâti à partir de l'unité de voisinage (jiefang), comme Nanyan fang, et un type d'implantation temporaire les cun, barraquement d'un étage, juste à côté des usines, en appui des villages, comme à Shenghuo cun, Qingshan (André, 2020a : 75-76, 201, 288-289).

Si en Chine, comme ailleurs en Asie, l'urgence est alors à la construction de logements pour améliorer les conditions de vie des populations urbaines, la Chine se démarque peu à peu par une priorisation donnée au desserrement industriel sur le résidentiel.

\section{2. Les villes satellites de desserrement industriel}

Le Ir $^{\text {rr }}$ plan quinquennal chinois (1953-1957), la collectivisation du foncier et la priorité donnée à l'industrialisation du pays confirment la cité ouvrière (gongren xincun) d'inspiration soviétique, comme choix d'aménagement résidentiel pour accompagner, objectif premier de la programmation économique, le développement industriel, en y hébergeant les travailleurs des manufactures adjacentes (Hoa, $1981: 63-65$; Petit, $2002:$ 81-86). Les premières « villes satellites " (weixingcheng) chinoises de desserrement industriel sont programmées à Pékin en 1953 et à Shanghai en 1956 et bénéficient de l'expérience d' "experts soviétiques", comme souligné dans les musées de l'urbanisme de ces deux municipalités. Dans le cadre du $I^{\mathrm{e}}$ plan quinquennal (1958-1962), les villes littorales chinoises sont l'objet d'un redéploiement industriel vers leurs périphéries; l'industrialisation s'accompagne d'un mouvement de desserrement industriel - et secondairement résidentiel. Ce programme de villes satellites se présente comme une alternative raisonnée au 
problème de croissance des très grandes villes. Sur le modèle du développement soviétique, il prévoit la délocalisation en périphérie des projets industriels de grande échelle (Kirkby, 1987 : 207). De nombreuses villes satellites industrielles, juxtaposées les unes aux autres, se développent alors autour de Wuhan, Zhengzhou, Hefei, Nankin, Shanghai, Tianjin, Pékin, Daqing Jinan, Handan, Changchun, Canton, Changsha (Funk, $1996: 324,338$ ). Cette première période de création de villes nouvelles correspond à la " phase d'industrialisation de la Chine sans extension urbaine » (Ma, 2002), et se traduit par le développement de villes satellites destinées à rationaliser et à optimiser le développement industriel soutenu. II s'agit par exemple des villes satellites dédiées à la sidérurgie comme Baoshanwei au nord-est de Shanghai ou Shijingshan à l'ouest de Pékin. Ces villes satellites sont construites en continuité avec les bourgs-centres administratifs gravitant autour de l'agglomération et s'appuyant sur des axes de transport, comme les voies ferrées et/ou les voies d'eau, comme Minhang à Shanghai prioritairement, et secondairement les routes principales. Autour de ces extensions mono-industrielles se juxtaposent, comme pour les villes nouvelles de l'Union soviétique et des pays socialistes européens, des zones résidentielles destinées aux travailleurs. Ces ensembles sont construits par le bureau de district ou d'arrondissement du travail, puis par les unités de travail (danwei), pour loger les ouvriers employés et leur famille, la main-d'œuvre nécessaire à l'effort d'industrialisation du pays (Photo 3 et 4). Peu à peu, la société chinoise, et par extension les villes chinoises, se transforment pour s'organiser autour et à partir des lieux de production. Un faible intérêt est alors porté au logement, aux équipements, à l'espace public et aux services urbains. Dans les faits, des migrations pendulaires des villes-centre vers les bourgs-centres sont attestés à Pékin, Shanghai, Wuhan. Ces villes satellites s'apparentent avant tout à des pôles de production industriels, implantés en périphérie de grandes villes. Sites industriels et cités ouvrières jouxtent alors les villages dédiés au maraîchage comme le prouve l'imbrication des tissus et des formes urbaines héritées de ces époques.

[Photo 3: Cités ouvrières dans l'ancienne ville satellite de Songjiang à Shanghai. 28 mai 2011, C. Henriot.]

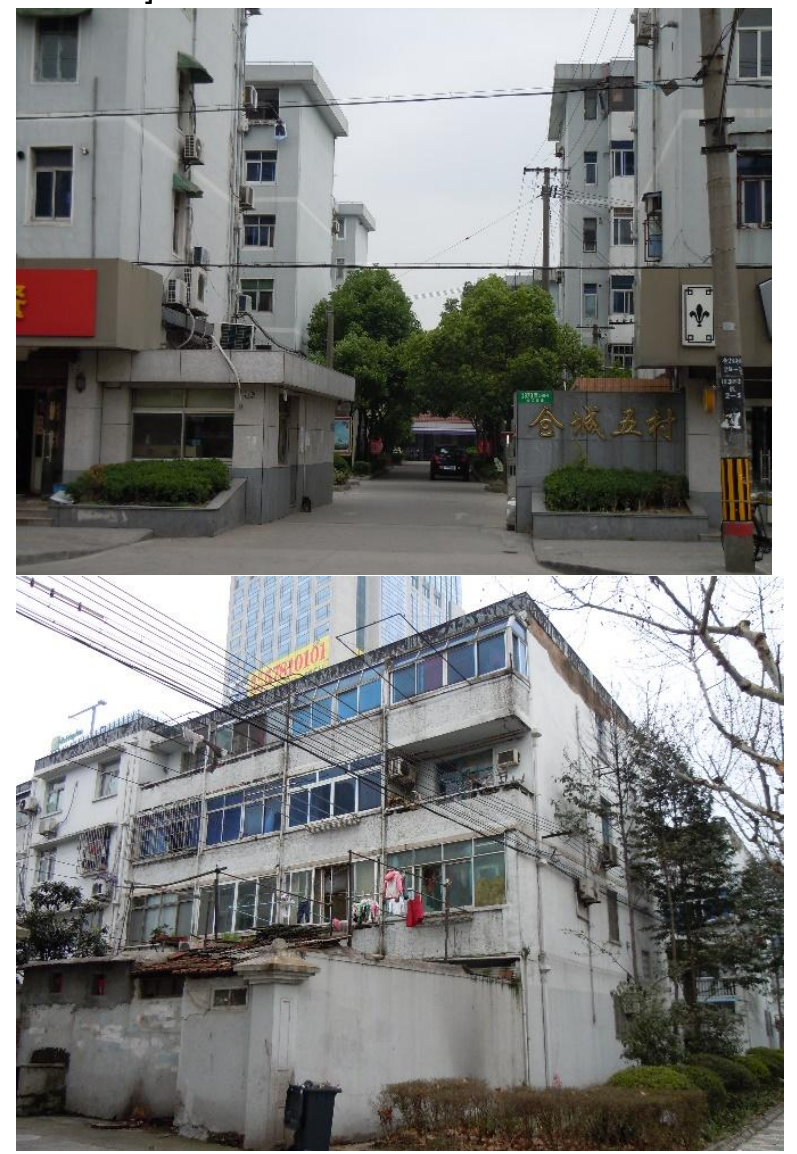


La réaffirmation de l'industrialisation rurale à partir de 1978 favorise le développement de ces villes satellites qui connaissent un nouvel essor dans les années 1980. Cette seconde phase de développement des villes satellites est cette fois portée par les aménageurs et urbanistes envoyés à la campagne pendant la Révolution culturelle et réhabilités au début des années 1980. Ces derniers reprennent les projets de déconcentration auxquels ils avaient participé vingt ans plus tôt (Kirkby, 1987: 223-224). Cependant, ils manquent d'expérience et de références pour améliorer les programmes. Les villes satellites doivent alors permettre la redistribution des populations qui reviennent de la campagne, où elles avaient été envoyées pendant la Révolution culturelle. Dans certains cas, leur retour est conditionné par un accord préalable : accepter de vivre et de travailler dans les complexes industriels, comme le complexe pétrochimique de Jinshanwei à Shanghai. Les villes satellites doivent également permettre de polariser et d'accueillir le surplus de main-d'œuvre agricole dégagé par les réformes agricoles.

Contrairement aux expériences menées en Asie orientale, la planification des villes satellites ne peut s'appuyer sur l'expérience internationale en raison de l'isolement relatif que connaît la Chine jusqu'à la fin des années 1970 (Hoa, 1981 : 63-65). Néanmoins, au bénéfice d'un renouveau des échanges, les projets chinois de villes nouvelles de desserrement métropolitain se rapprochent des expériences régionales et internationales à la fin du vingtième siècle.

\section{3. Les villes nouvelles de desserrement métropolitain}

Au Japon, dans les années 1970, soit vingt ans après l'expérience monofonctionnelle des quartiers résidentiels satellites, les projets de villes nouvelles s'étoffent. En 1972, est adoptée une " charte pour le développement des villes nouvelles " (Shi-toshi kiban seibi ho) qui se réfère au New Town Act britannique de 1946 (Nagamine, 1987). Il s'agit d'une politique nationale de villes nouvelles. À Hong Kong, dans les années 1980, une véritable politique de décentralisation et d'aménagement d'ensemble du territoire de Hong Kong permet un redéploiement de la population et des activités du cœur Kowloon-Hong Kong vers les Nouveaux Territoires (Yeh, 1987) et ses villes nouvelles. En Corée du Sud, si dans les années 1980, des "cités neuves" (sin sigaji) à la fonction résidentielle prédominante, ressemblent aux zones à urbaniser en priorité (ZUP) françaises; dans les années 1990, un programme de "villes nouvelles " ( $\sin$ doji) faisant explicitement référence au modèle européen, notamment français est implanté (Gélézeau, 2003 : 72 ; Gélézeau, 2011 : 58-59).

En Chine, les pratiques d'urbanisme héritées du système de planification quinquennale sinosoviétique évoluent peu à peu : dès le début des années 1980 à Pékin ou en Chine du Sud, au début des années 1990 à Shanghai, et seulement au milieu des années 1990 ailleurs dans le pays. Dans les années 1990, les projets de développement des grandes agglomérations chinoises adaptent le modèle des villes-satellites dans un contexte d'ouverture à l'expertise internationale, de réouverture du marché de l'immobilier et de modernisation urbaine. La gouvernance métropolitaine est désormais assurée par les gouvernements municipaux, tandis que les milieux d'affaires investissent la production urbaine (Lin et Wei, 2002 : 1537-1538). Certaines métropoles littorales bénéficient d'une concentration de moyens financiers, techniques et humains, sous l'impulsion de nouveaux acteurs, comme les bailleurs de fonds internationaux, les cabinets d'architecture-urbanisme internationaux, les investisseurs Chinois de l'Outre-mer, qui participent à la circulation de modèles urbains internationaux et véhiculent une nouvelle idée de la ville chinoise moderne et globalisée.

Par exemple, si dès 1984, la mission interministérielle de l'information scientifique et technique (MINIST) française, accompagnée de l'Institut d'aménagement et d'urbanisme de la région île-deFrance (IAURIF), en charge de la veille et de la prospective pour les villes nouvelles franciliennes, rencontrent les instituts d'information scientifique et technique des municipalités de Pékin et de Shanghai (Henry, 1984: 65-70), il faut attendre la fin des années 1980, pour que des échanges réguliers, bien que difficiles, soient entretenus entre I'IAURIF et les responsables en charge de la 
planification des villes-satellites de Shanghai (Antier et Abadia, 1987 ; Antier, 1989) et de Pékin (Etteinger, 1992 ; Larivière, 1997). Au début des années 1990, les premiers cabinets d'architecture et d'urbanisme étrangers s'implantent en Chine, comme les cabinets français Arté-Charpentier ou ADP Paul Andreu. Ces acteurs français, publics et privés, en mobilisant l'expérience des villes nouvelles françaises, participent à la transmission du modèle français de villes nouvelles et permettent aux acteurs chinois de se réapproprier les schémas mais aussi les outils de l'urbanisme réglementaire, la planification et l'urbanisme opérationnel (Petit, 2002 : 21-43).

L'arrivée des opérateurs internationaux entraîne des ajustements structurels, favorise une gestion urbaine décentralisée et de nouvelles formes de planification dominées par la logique de projet, notamment dans les grands équipements, les services et les réseaux urbains (Lorrain, 2010 ; Lorrain 2011 ; Zhang et al., 2014 ; Zhang et al., 2015).

Les villes nouvelles chinoise construites, dans les années 1990, par exemple à Yizhuang au sud-est de Pékin, sur l'axe Pékin-Tianjin (Larivière, 1997), dans les années 2000, par exemple Tongzhou à Pékin (Bonino et al., 2019 ; Fiandanese, 2019), par exemple Jiading, Songjiang (cf photo 5) et Qingpu à Shanghai (Henriot, 2013 ; Liu, 2014), par exemple Zhengdong à Zhengzhou ( $c f$ photo 6) sont de véritables pôles multifonctionnels de desserrement, de différenciation et de spécialisation dans la municipalité. Ils adossent à des polarités rurales anciennes, voire des villes-satellites, de vastes secteurs aménagés suivant les principes du fonctionnalisme introduits dans les années 1990 (Zhuo, 2004; Liu, 2018), qualifié également d'" hyper-fonctionnalisme" (Curien, 2014), ou de "modèle intégrationniste " (Doulet, 2015), couplés au principe du transit-oriented development (TOD) (Delpirou, Doulet, Zhuo, 2015; Zhuo, 2015; Shen et Wu, 2019; André, 2020a). Toutefois, contrairement au modèle collectiviste des unités de travail, qui associait sur un même lot l'usine, les logements du personnel et l'ensemble des commerces et services destinés aux habitants, la différenciation des espaces et leur spécialisation fonctionnelle induisent des déplacements et des mobilités croissantes : les distance-temps ne cessent de croître. La rationalisation de la trame et du tissu urbain inscrit le zonage fonctionnel et les nouvelles mobilités générées dans un plan orthogonal orienté nord-sud et est-ouest ( $c f$ photo 7 ). De larges artères se coupant à angle droit et dont le gabarit répond aux nouvelles règles d'urbanisme, séparent strictement les usagers (quatre roues/deux roues/piétons) donnant incontestablement l'avantage à l'automobile (cf photo 8). L'ensemble articule de vastes secteurs résidentiels de grands collectifs verticalisés ( $c f$ photo 9 ), de petits collectifs en bande, du pavillonnaire individuel, des antennes universitaires déconcentrées, des parcs d'agrément, des centres commerciaux, ainsi que des zones industrielles et commerciales périphériques. Ces villes nouvelles sont reliées à la ville-centre par un réseau dense d'autoroutes et un réseau ferré léger, comparable au RER francilien ( $c f$ photo 10 ) et certaines connaissent même l'arrivée du tramway ( $c f$ photo 11). Wuhan développe d'ailleurs son propre modèle de planification orienté par/vers le réseau ferré léger : le " bourg-métro " (André, 2020b : 166). Ce zonage aménage une ville souhaitée plus efficace dans son fonctionnement. Ainsi les planifications urbaine, foncière et la planification des transports fusionnent dès 2009 à Shanghai (Henriot, 2013). II faut attendre le milieu des années 2010 à Wuhan (André, 2016, 2020a).

Ces villes nouvelles correspondent à des opérations très lucratives de valorisation du foncier, aménagées par de nouveaux acteurs de la production urbaine : les "sociétés de développement " (chengshi touzi kaifa gongsi) qui œuvrent dans l'intérêt du gouvernement d'arrondissement (Li et Chiu, 2018; Shen et Wu, 2017). Les opérations immobilières des villes nouvelles sont orientées vers les segments solvables de la population -les classes moyennes émergentes (Henriot, 2013a), tandis que les nouveaux quartiers de logements sociaux sont relayés en marge des périmètres d'intervention, voire planifiés en dehors des secteurs de villes nouvelles (Henriot, 2014).

Ces nouvelles entités urbaines doivent participer au développement d'ensemble de la région métropolitaine. Elles visent une certaine autonomie à l'échelle de la ville nouvelle (écoles, hôpitaux), 
tout en s'inscrivant en complémentarité fonctionnelle et morphologique à l'échelle de l'aire métropolitaine (antennes universitaires déconcentrées, zones d'activités, parcs de récréation, offre résidentielle diversifiée). Initialement conçues comme des espaces de desserrement des agglomérations et de rationalisation de leurs tensions périphériques; elles participent à la fois à la redistribution des activités et à celle de la population (Henriot, 2013b : 171-201; Henriot, 2016).

[Photo 5 : La maquette de la ville nouvelle de Songjiang à Shanghai présente une vraie diversité des formes urbaines. 23 août 2016, C. Henriot.

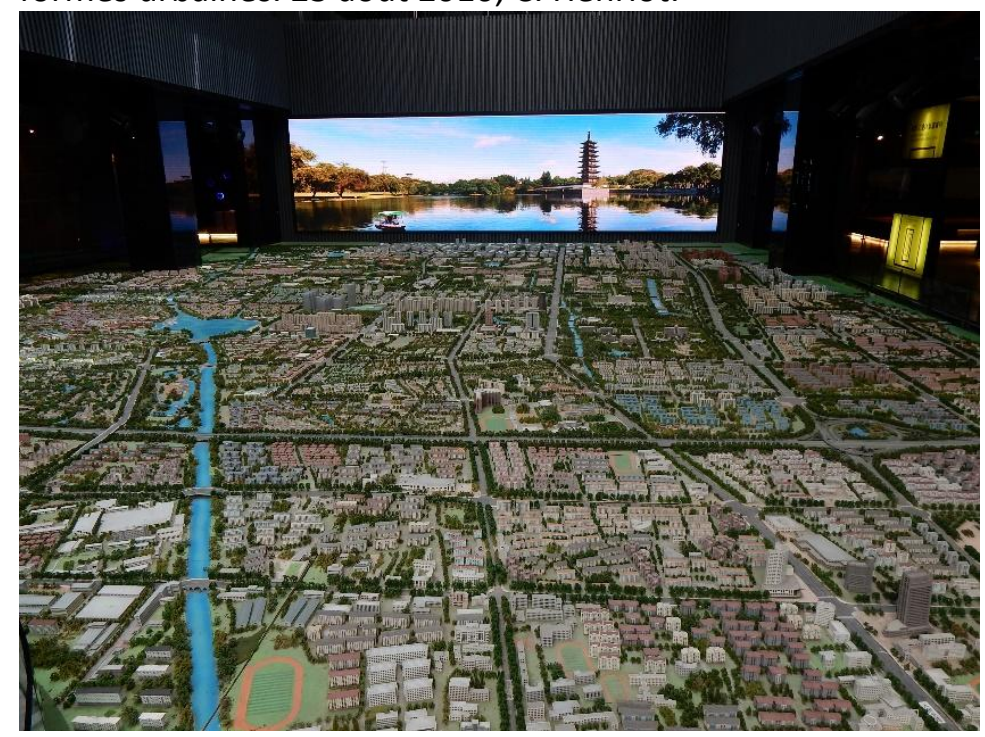

[Photo 6 : Le cœur de la ville nouvelle de Zhengdong à Zhengzhou. 5 mai 2007, C. Henriot.]

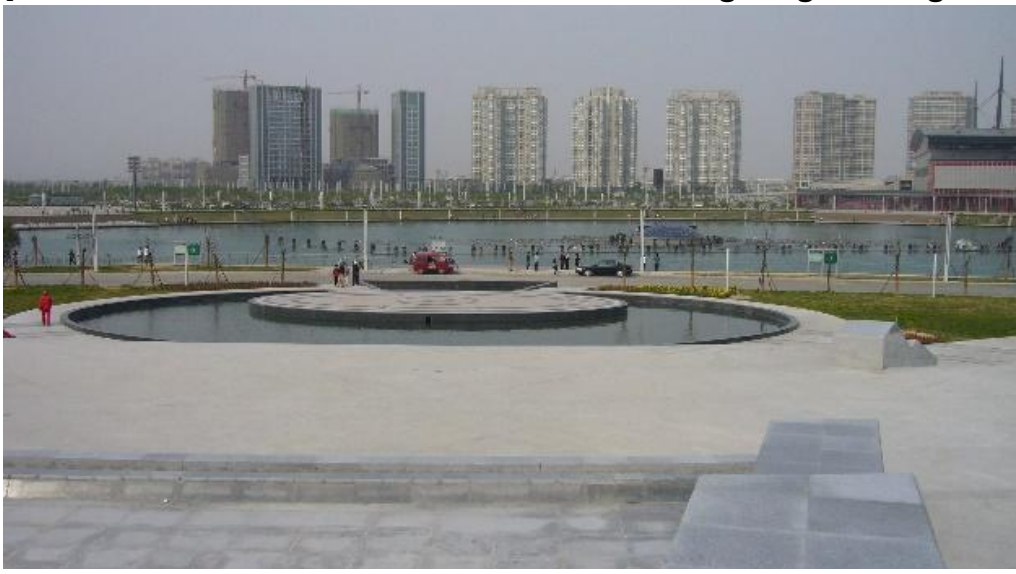

[Photo 7: Les villes nouvelles sont planifiées sur une trame orthogonale de larges avenues quadrangulaires, Songjiang à Shanghai. 5 avril 2011, C. Henriot.] 


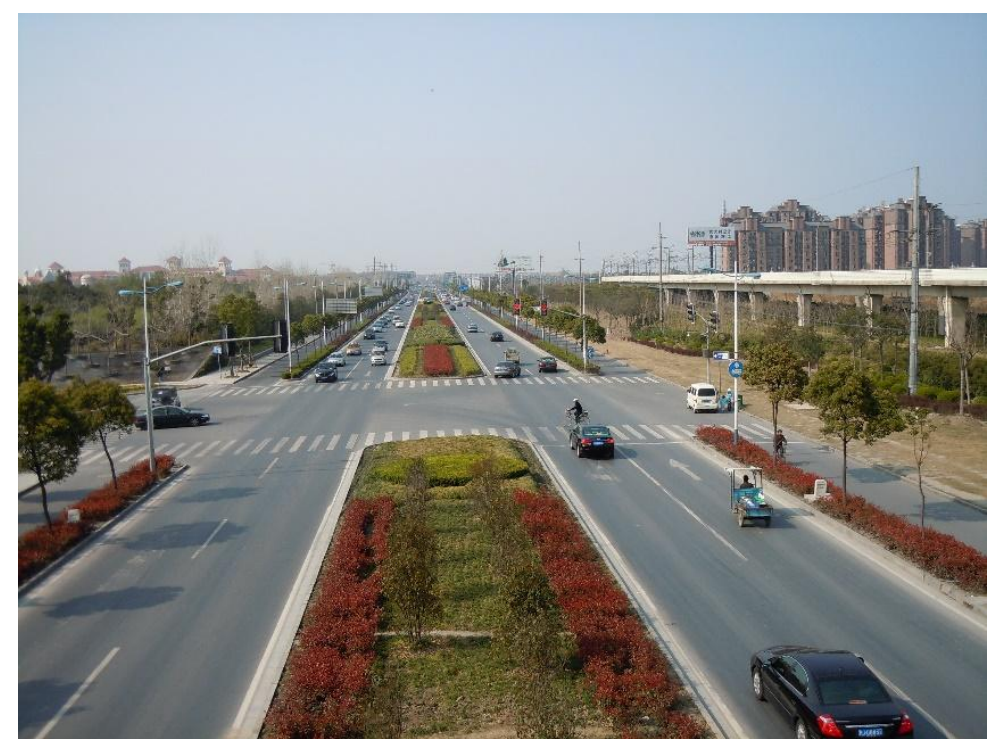

[Photo 8: La planification traditionnelle accorde une place importante à la voiture, Songjiang à Shanghai. 22 août 2016, C. Henriot.]

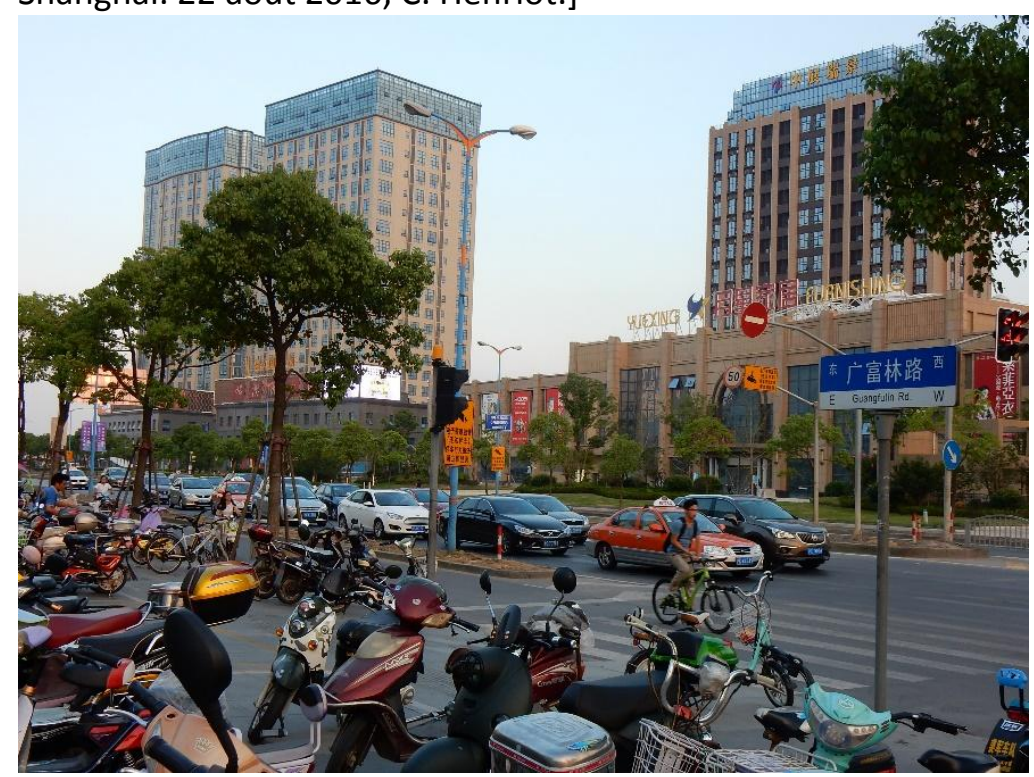

[Photo 9 : Un résidence verticalisée à Songjiang, Shanghai. 22 août 2016, C. Henriot.]

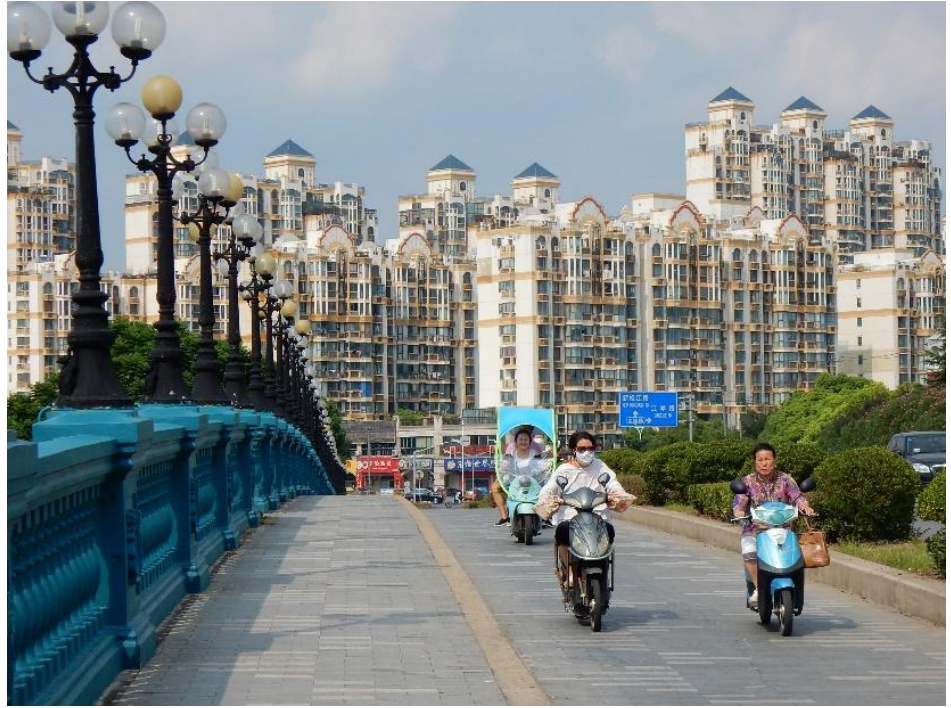


[Photo 10: Le métro aérien et surélevé en banlieue Métro ligne 11, ville nouvelle de Jiading, Shanghai. 30 mai 2011, C. Henriot.]

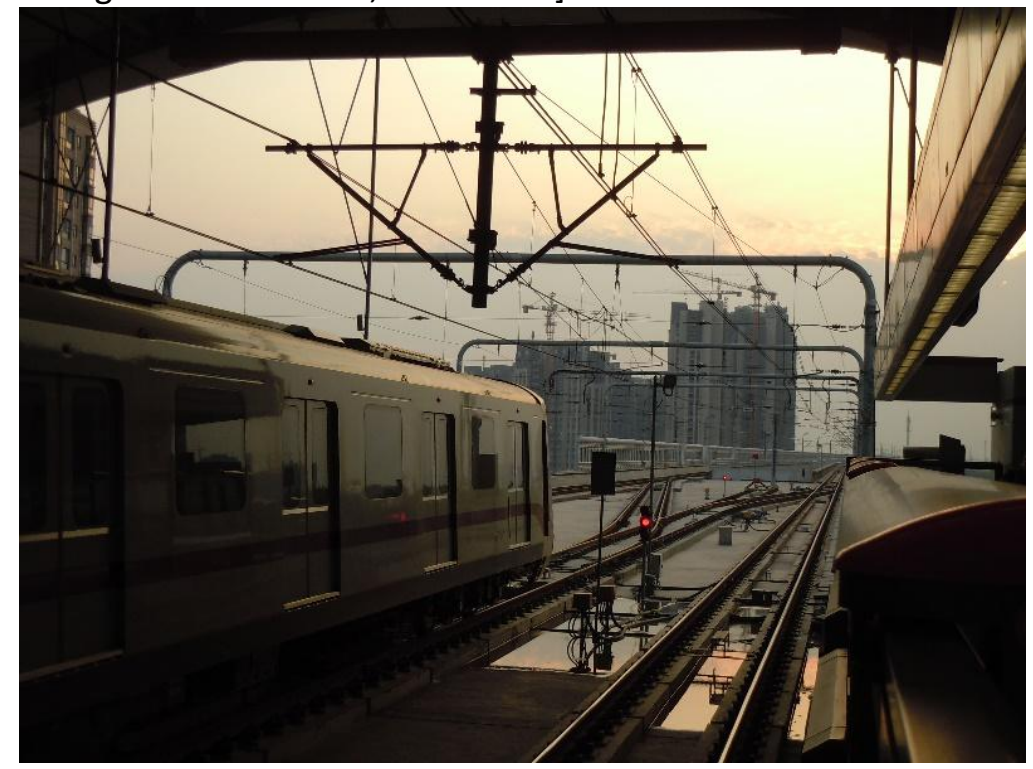

[Photo 11 : Le tramway, au pied du métro aérien et surélevé en banlieue, Métro ligne 7, ville nouvelle de Songjiang, Shanghai. 30 mars 2019, C. Henriot.]

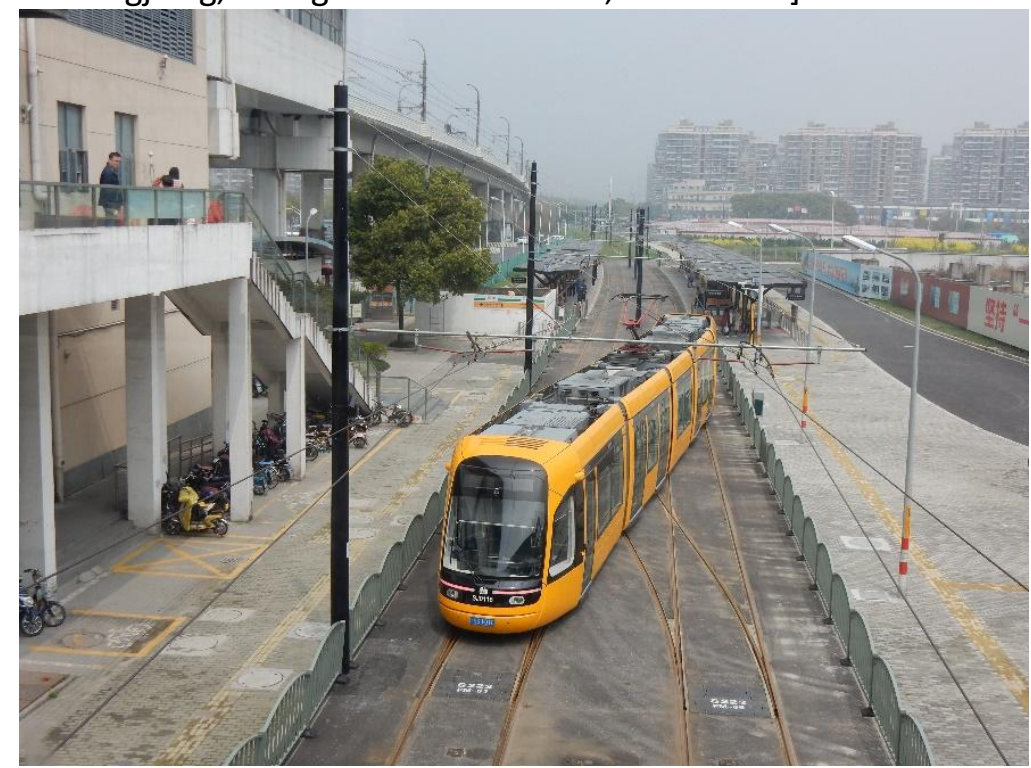

Ainsi ces villes satellites et ces villes nouvelles évoluent-elles entre la fin des années 1950 et nos jours, en fonction des réponses qu'elles sont censées apporter en termes d'urbanisme et d'aménagement du territoire chinois. Ces deux figures de la New town, portées par la planification municipale, sont intimement liées à la trajectoire urbaine chinoise, laquelle promeut, dans un premier temps, un desserrement industriel grâce aux villes satellites d'influence soviétiques ou sinosoviétique. Puis, l'ouverture à la planification fonctionnaliste traditionnelle renouvelle la planification des villes nouvelles qui deviennent de véritables pôles multifonctionnels de redéploiement métropolitain, visant à répondre aux objectifs quantitatifs d'urbanisation. Au début du vingt-etunième siècle, la montée des orientations environnementale et numérique participe d'un renouvellement de la figure de la ville nouvelle chinoise. 

urbanisation qualitative?

Au milieu des années 2000, la Chine connaît une intégration accélérée à la globalisation et s'insère sur la scène internationale ; les villes chinoises se modernisent, ce qui se traduit par des inflexions de l'action publique urbaine qui s'intéresse successivement aux paradigmes du nouvel urbanisme (new urbanism) et de mondialisation, de développement durable et de ville intelligente. Nous faisons l'hypothèse que les villes nouvelles thématiques, les "nouveaux bourgs " d'architecture néotraditionnelle occidentale ou néo-chinoise, les "villes durables" et les "villes intelligentes" renvoient à une même volonté politique d'orienter la croissance urbaine vers un développement maîtrisé, répondant à une certaine standardisation internationale, en reproduisant et en adaptant par un arrimage local certains modèles urbains contemporains, afin de diversifier et améliorer la production urbaine chinoise. Ces trois avatars de la ville nouvelle chinoise contemporaine témoigneraient ainsi d'une "mondialisation proprement chinoise » (Sanjuan, 2004: 16-17; Friedmann, 2006) de la ville nouvelle.

\section{III.1. Les « nouveaux bourgs », entre nouvel urbanisme et architecture néo-traditionnelle}

Le début des années 2000 est marqué par un engouement certain pour la construction de quartiers résidentiels d'architecture occidentale : français, anglais, allemand, italien, américain, visant pour les promoteurs à se distinguer sur le secteur résidentiel devenu très concurrentiel et réservé à des populations aisées à très aisées. Au-delà de cette distinction immobilière, plusieurs programmes pilotes de "nouveaux bourgs " relevant tantôt du nouvel urbanisme, tantôt de l'architecture néotraditionnelle occidentale ou chinoise ont été planifiés.

Ces nouveaux bourgs présentent pour dénominateur commun un renouvellement l'urbanisme chinois. La planification traditionnelle, les vastes îlots quadrangulaires et le zonage strict des villes nouvelles s'effacent, localement (de manière très localisée), pour laisser s'exprimer les principes de conception urbaine du nouvel urbanisme et de l'architecture néo-traditionnelle occidentale ou chinoise : une échelle d'aménagement, une densité urbaine, des espaces publics, un rapport entre le bâti et les vides qui soient favorables aux mobilités piétonnes et aux activités communautaires. À Shanghai, par exemple, le projet " une ville, neuf bourgs " (yi cheng jiu zhen) lancé en 2001, planifie l'aménagement de bourgs d'architecture occidentale en périphérie de Shanghai (Wang et Li, 2006 ; Yu et Zong, 2009; Nieuwenhuis, $2010 ; \mathrm{Li}, 2010)$. Ils proposent le retour à une diversité morphologique, fonctionnelle et sociale des quartiers, ainsi qu'aux mobilités douces - en utilisant comme éléments de discours des références à la mondialisation, mais aussi des références à des projets de villes nouvelles relevant du mouvement des New Towns. En effet, dans le cadre du Xe plan quinquennal (2001-2005), dont le principal slogan est d'« urbaniser la banlieue », le gouvernement central publie, fin 2000, un document promouvant le développement des unités urbaines de petite et de moyenne dimensions. En réponse, la municipalité de Shanghai livre en janvier 2001, des "Propositions pour développer les bourgs de Shanghai », à l'origine du projet pilote " une ville, neuf bourgs ". Chaque arrondissement de banlieue est invité, par la Municipalité, à développer sur son territoire un « bourg pilote» (shidian zhen).

Dans leur conception, les schémas directeurs mobilisent le modèle de la cité-jardin d'Ebenezer Howard pour favoriser l'équilibre entre le centre et chaque cité-jardin, l'autonomie à l'échelle de la cité-jardin, et l'intégration de la ville à la campagne. L'aménagement d'ensemble doit favoriser les espaces publics, les espaces verts et l'eau comme éléments structurants du paysage urbain pour créer une centralité urbaine dédiée aux mobilités douces (Wang et Li, 2006 ; Zhou et Xue, 2008). La référence internationale la plus mobilisée pour porter ce projet auprès des aménageurs et décideurs 
politiques shanghaiens est d'ailleurs Milton Keynes ${ }^{3}$. Chaque projet doit en outre proposer une alternative au développement immobilier naissant, qui, pour minimiser les coûts et rationaliser la production, réplique le même bâtiment à grande échelle, ce qui crée un paysage résidentiel très homogène au sein d'une même parcelle et des ruptures morphologiques très importantes entre parcelles. Pour pallier cette homogénéité, le document municipal de 2001 attire l'attention des gouvernements locaux sur l'insertion de la Chine dans la mondialisation et sur la nécessité d'améliorer la forme et le fonctionnement des villes chinoises, en s'appuyant sur des styles architecturaux diversifiés. Pour mieux identifier les banlieues, chaque bourg nouveau du programme " une ville, neuf bourgs " présente un "style caractéristique " (tese fengmao), souvent allogène, selon le principe "une ville, un style" (yi cheng yi mao) (Zhou et Xue, 2008). Ces bourgs correspondent à de petites unités urbaines dont la trame viaire est aisément identifiable et s'inscrit en discontinuité par rapport à la trame orthogonale qui structure souvent les villes chinoises contemporaines. Cette diversification morphologique permet également d'améliorer l'image de la banlieue auprès des populations du centre-ville de Shanghai. Dix bourgs ou villes nouvelles à la morphologie urbaine et à l'architecture originales sont ainsi construits à Shanghai entre 2003 et 2006. Certains projets urbains relèvent du nouvel urbanisme, comme le nouveau bourg de Anting ( $c f$ photos 12 et 13), comme le bourg de Zhujiajiao, comme le bourg de Pujiang, dessiné par Gregotti, en s'inspirant de l'espace public des villes traditionnelles italiennes (cf photo 14), tandis que d'autres projets relèvent de l'architecture néo-traditionnelle occidentale, comme "le bourg sur la Tamise", Thames Town, « un cliché à l'anglaise » (Henriot et Minost, 2017 ; Piazzoni, Banerjee, 2018) (cf photo 15 et 16), comme la ville scandinave de Luodian, ou la ville hollandaise de Gaoqiao.

[Photo 12 : La maquette du bourg de Anting, Shanghai. 30 mai 2011, C. Henriot.]

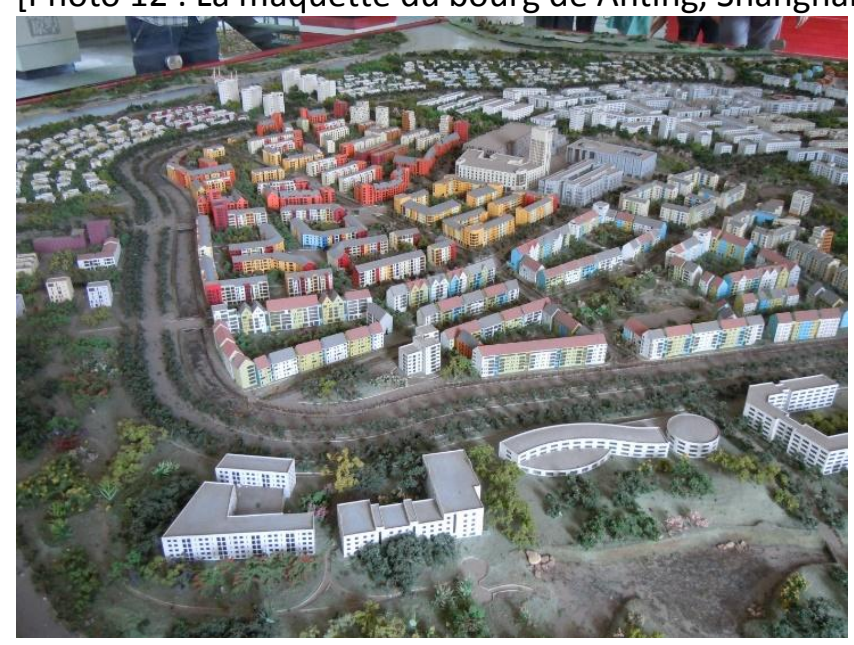

[Photo 13 : L'habitat individuel en bande sur les périphéries de Anting, Shanghai. 11 avril 2011, C. Henriot.]

\footnotetext{
${ }^{3}$ Milton Keynes est une ville nouvelle britannique, construite à partir de 1967, dans la périphérie de Buckingham. Elle présente une architecture innovante et promeut les premiers bâtiments sobres en énergie. Les décideurs politiques chinois connaissent et mobilisent cet exemple pour faire référence à une ville nouvelle innovante.
} 


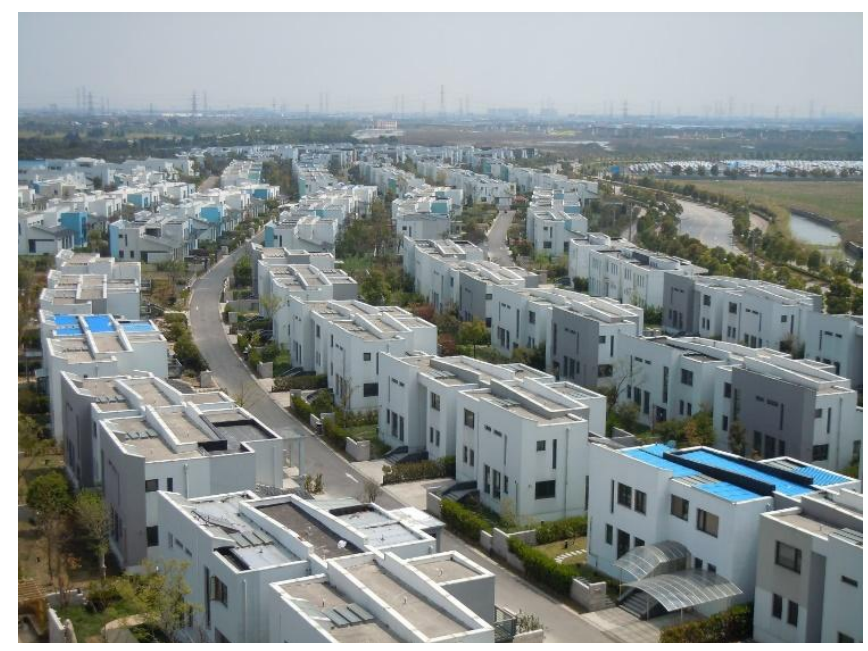

[Photo 14 : Le nouveau bourg de Pujiang, Shanghai. 26 février 2011, C. Henriot.]

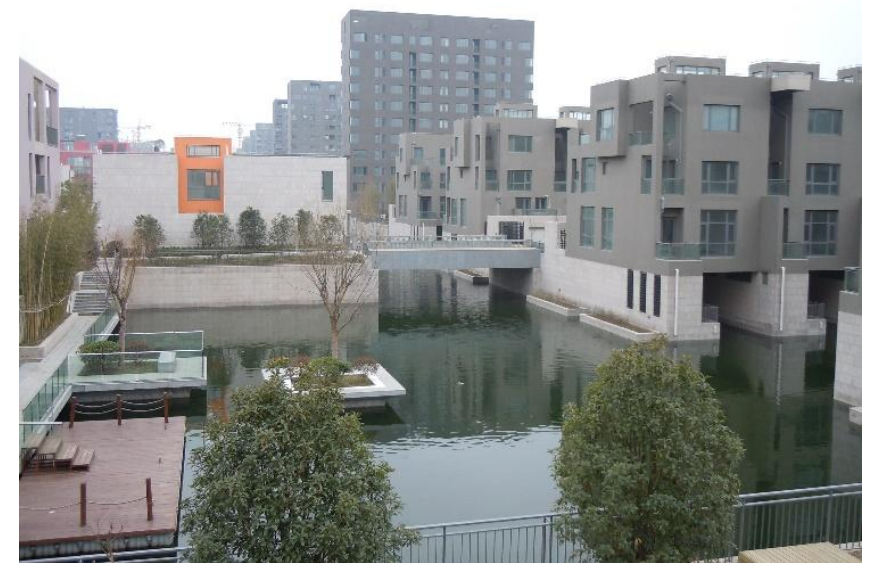

[Photo 15 : La maquette de Thames Town, dans la ville nouvelle de Songjiang, Shanghai. 23 août 2016, C. Henriot.]

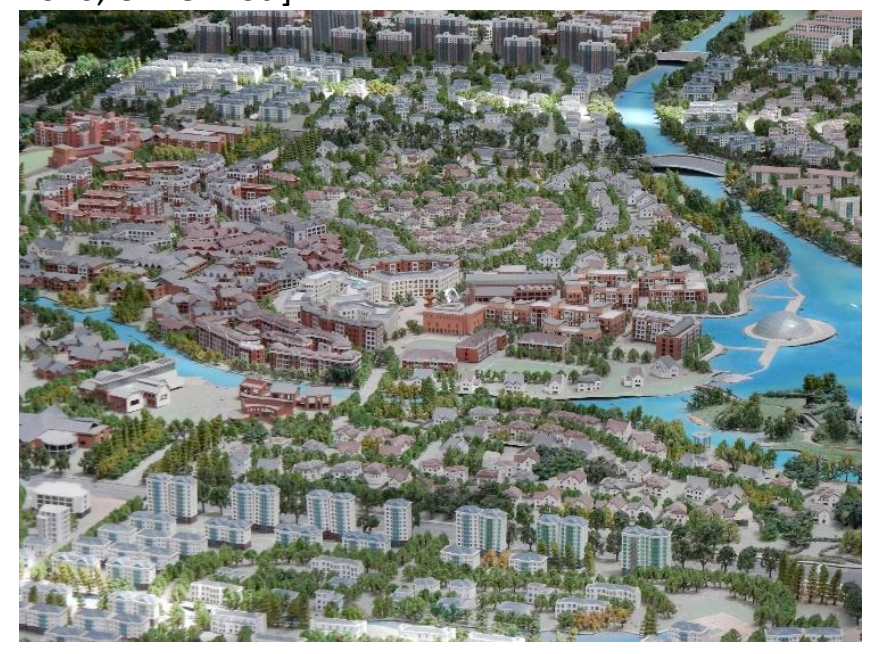

[Photo 16 : Le centre bourg de Thames Town et ses architectures néo traditionnelles à l'anglaise. 30 mars 2019, C. Henriot.] 


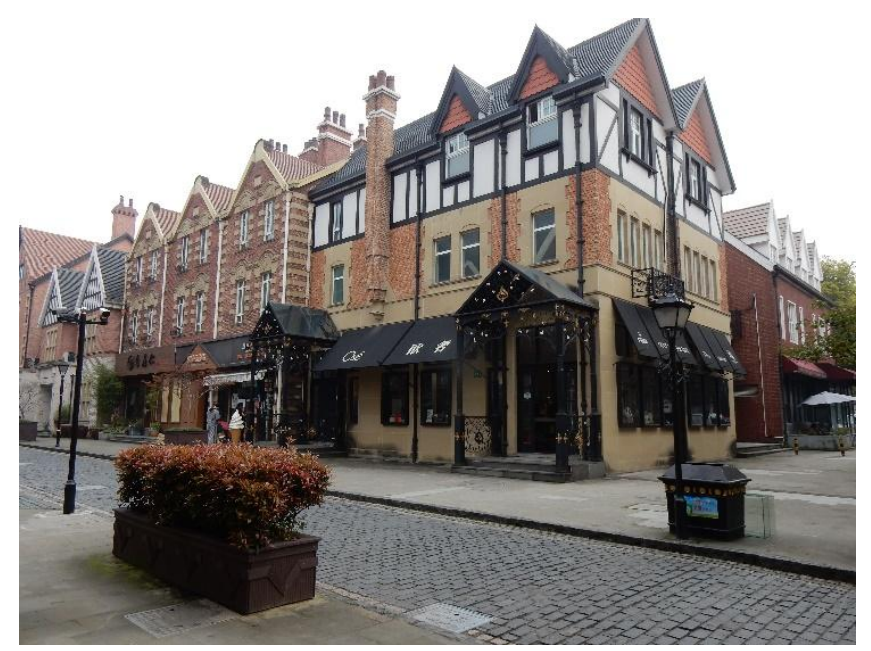

Les bourgs nouveaux construits au début du XXI ${ }^{\mathrm{e}}$ siècle rompent avec l'échelle d'aménagement et le fonctionnalisme des villes nouvelles, pour répondre à des objectifs d'urbanisation qualitatifs - tout comme les inflexions thématiques de la ville durable et de la ville intelligente.

\section{III.2. Les villes nouvelles durables, un tournant environnemental limité ?}

Si la question environnementale émerge dans le champ de l'urbanisme à partir de 1992, la Chine engage un véritable " tournant environnemental » (Curien, 2017) dans la seconde moitié des années 2000. Dans le cadre du XXI ${ }^{\mathrm{e}}$ plan quinquennal (2006-2010), les politiques de développement urbain sont infléchies pour désormais prendre en considération l'environnement, le développement urbain durable et la lutte contre le changement climatique.

Le gouvernement central nourrit alors pour ambition d'élaborer un modèle chinois de ville durable : le paradigme de l'éco-construction est exploré par le ministère du Logement et du développement urbain et rural à travers le label " éco-cité-jardin " (shengtai yuanlin chengshi), initié en 2004 et dont les projets pilotes sont identifiées en 2006, celui de la ville verte est privilégié par le ministère de la Protection environnementale avec le label " éco-ville " (shengtai chengshi) en 2007, tandis que celui de la "ville bas-carbone" (ditan chengshi) est promu par la Commission nationale pour le développement et les réformes à partir de 2010 (Baeumler et al., 2012 ; Zhou et al., 2012 ; Henriot, 2013b : 81-86; Henriot, 2015). La Chine encourage également le développement d'un quatrième type de villes durables, portées par la coopération internationale et la coopération bilatérale. Les projets conçus dans le cadre de partenariats internationaux sont ambitieux et largement médiatisés. Toutefois, il convient de se montrer prudent quant à la durabilité de ces projets.

En effet, si la ville écologique sino-singapourienne de Tianjin, "Sino-Singapour Tianjin Eco-City " (SSTEC), lancée en novembre 2007 à des fins économiques, fait l'objet d'évaluations positives par les bailleurs de fonds comme la Banque mondiale (2009) ou la Banque asiatique de développement (2010), l'initiative Sino-Singapourienne développée à Suzhou, le "Suzhou Industrial Park " (SIP) est qualifié de planification urbaine "hyper-productiviste et fonctionnaliste " éloignée des préoccupations environnementales (Curien, 2014; Curien, 2017: 32). L'expérimentation sinosingapourienne de la SSTEC constitue quant à elle une référence pour sa planification et son aménagement intégrés des services essentiels, mais reste focalisée sur la technologie et l'infrastructure, et surtout conserve la métrique des projets fonctionnalistes traditionnels (Curien, 2017 : 35). Si la construction d'indicateurs de performance était censée permettre l'évaluation du dispositif de la SSTEC mais également sa diffusion ( $\mathrm{Li}$ et al, 2018), cette production urbaine intégrationniste des services essentiels reste exceptionnelle, extraordinaire au regard de la production urbaine chinoise. Ces villes nouvelles sino-singapouriennes, portées dans le cadre d'une 
politique bilatérale, bénéficient d'une concentration d'expertise, d'une collusion d'intérêts entre hauts-dirigeants chinois et singapouriens qui ne peuvent absolument pas être répliqués ailleurs sur le territoire chinois.

Il en va de même à Wuhan, où la France est associée, dans le cadre d'une coopération bilatérale ancienne, par les autorités provinciales et municipales chinoises, au projet de ville durable à Wuhan à la planification de l'éco-ville sino-française de Caidian, déclinaison du modèle chinois de "ville éponge " (haimian chengshi) (André, 2020a : 172-178; 189-191). Il est évident que cette production urbaine durable, conçue dans le cadre de projets urbains internationaux, échappe à la ville nouvelle de desserrement métropolitain conçue dans le cadre de politiques municipales de redéploiement fonctionnel.

Si la figure de la ville nouvelle de desserrement municipal relève, en Chine comme ailleurs, d'une production urbaine particulière, ces projets urbains de ville durable nourris par des coopérations internationales s'apparentent à une production urbaine extraordinaire, au sens de non-ordinaire. La ville nouvelle durable ordinaire chinoise ne peut être, à ce point, exemplifiée. Son projet urbain apparaît dégradé, voire déclassé. Il s'agit par exemple de l'éco-cité de Dongtan à Shanghai. Dongtan était initialement un projet de coopération internationale, une éco-ville sino-britannique qui a fait long feu, en raison de la volatilité des élites (Henriot, 2015). Du projet initial ne subsiste que le Parc national de récréation de la zone humide de Dongtan à l'extrémité sud-est de l'île de Chongming, tandis qu'une partie des secteurs qui devaient être aménagés dans le cadre de la ville nouvelle écologique a bien été ouvert à la promotion immobilière résidentielle orientée vers le segment du luxe, via la commercialisation de maisons individuelles.

Les rares villes nouvelles durables chinoises, lesquelles semblent systématiquement s'inscrire dans un projet de coopération bilatéral, ne rompent pas avec les principes d'aménagement fonctionnalistes, mais constituent de véritables laboratoires de la production urbaine " extraordinaire »- comme, pour d'autres raisons, les villes intelligentes.

\section{III.3. Des villes intelligentes pour renouveler la production urbaine ?}

À la fin des années 2000, la " ville intelligente " chinoise naît dans un contexte de crise économique entraînant une chute des exportations chinoises, un repli vers le marché intérieur, et d'importants investissements en infrastructures et dans l'internet des objets (Wu et al, 2018), orchestrés par le gouvernement central (Lu et al. 2015). Ce nouveau mode de production de la ville, développé en appui de la ville existante, comme une " couche » numérique, en renouvellement urbain, comme en extension urbaine, supplante la ville durable parmi les avatars de la ville du futur (Douay et Henriot, 2016 ; Henriot, 2018). La dimension expérimentale et le renouvellement des usages induits par ces nouveaux dispositifs technologiques nous invitent à considérer la ville intelligente comme une inflexion de la ville nouvelle.

La notion de ville intelligente et ses perspectives de développement économique sont rapidement apprivoisées par l'Etat central chinois. Comme nous l'avons déjà souligné (Henriot et al., 2018), la NDRC et au-delà le gouvernement central nourrissent pour ambition d'élaborer un modèle chinois de ville intelligente. Ils mobilisent pour cela l'ensemble des acteurs à leur disposition en les positionnant en situation de concurrence. Ainsi les initiatives se multiplient-elles. Un premier projet de coopération entre le ministère chinois de l'Industrie et des Technologies de l'Information (Ministry of Industry and Information Technology - MIIT et la Direction générale des Réseaux de Communication, du Contenu et des Technologies (CNECT) de la Commission européenne établit un référentiel sinoeuropéen d'expertise sur la ville intelligente pour favoriser la conception de "Green Smart City " sur 15 sites pilotes (EU-China, 2014). Dès 2012, le MIIT et la Commission pour les réformes et le développement (National Development and Reform Commission - NDRC) conçoivent et diffusent un 
document national promouvant le développement de villes intelligentes. Un avis est formulé quant à la réalisation de sites pilotes nationaux de villes intelligentes, avec la mise en avant de mesures provisoires et transitoires, pour démarrer les applications concrètes à l'échelle des districts et des municipalités. Le MIIT choisit de développer deux sites pilotes en Chine de l'Est: Yangzhou et Changzhou. Parallèlement, le ministère pour la Science et la Technologie (Ministry of Science and Technology - MOST) organise, en 2012, une alliance dédiée aux villes intelligentes, la " China Smart City Industry Alliance ", et promeut deux sites pilotes: Shenzhen et Wuhan. Cependant, l'ensemble de ces sites pilotes relèvent d'une production urbaine extraordinaire.

Pour encourager une production urbaine ordinaire qui suive les préceptes de la ville intelligente, en 2012, 2013 et 2014, trois appels d'offres successifs invitent les collectivités territoriales chinoises à présenter des projets leur permettant d'être labellisées comme site pilote de "ville intelligente " (zhihui chengshi) par le ministère en charge des questions d'aménagement: le ministère du Logement et du Développement urbain et rural (Ministry of Housing and Urban-Rural Development MOHURD). À noter qu'en août 2014, la troisième phase de programmation de villes intelligentes correspond à une action commune au MOHURD et au MOST visant à regrouper les expertises des deux ministères. La mise en œuvre s'appuie sur l'implantation, à Pékin, d'un nouveau Centre national pour l'Innovation, mais fait aussi appel à la mobilisation de différents acteurs au niveau local, notamment pour financer ces projets par le partenariat public-privé. Faisant suite à ces appels d'offres, 290 démonstrateurs sont labellisés et encouragés. Au-delà, on estime à plus de 800 les initiatives smart sur le territoire chinois. Ce programme vise à diffuser l'internet des objets, le big data, l'informatique dans les nuages, la téléphonie mobile ainsi que l'intelligence artificielle dans les mégapoles, les grandes villes, mais également les villes moyennes chinoises, et ce sur l'ensemble du territoire ; à renforcer la capacité de planification des villes-centre au regard de leur périphérie; à promouvoir les TIC comme outil de gestion urbaine. Ces nouveaux dispositifs technologiques, ne se substituent pas à la production urbaine fonctionnaliste traditionnelle, ils l'enrichissent et renouvellent profondément les pratiques de la ville. Ce tournant smart est certainement le mode de production urbaine qui s'est le plus enrichi, diffusé et enraciné en Chine ces dernières années, encouragé par l'engouement des populations chinoises pour le numérique et poussé par les dirigeants politiques qui avaient autant à cœur les perspectives de développement économique que les capacités de contrôle des populations.

Certains projets de villes intelligentes restent discrets, se limitant à la généralisation de la vidéosurveillance des " communautés", d'autres projets sont particulièrement ambitieux, comme le confirme le cas de Hangzhou, où, conjointement avec la municipalité de Hangzhou, l'entreprise Alibaba, a implanté un " cerveau » à la ville, via l'« Alibaba Cloud» (ali yun), également appelé le " cerveau de la ville " (chengshi danao). Dès lors, Hangzhou " incarne " la ville intelligente (Henriot, 2018). Par ailleurs, l'actualité de la Covid-19, la nécessaire distanciation sociale, l'importance du contrôle des populations et l'injonction au traçage viennent renforcer le rôle stratégique que les autorités chinoises accordent à cette orientation numérique et optimisée de la fabrique de l'urbanisme chinois.

Ainsi, la succession de ces modèles thématiques de villes nouvelles (le "nouveau bourg " d'urbanisme nouveau ou d'architecture néo-traditionnelle, la ville durable, la ville intelligente) témoignent d'une seconde mondialisation de la ville nouvelle, de la circulation et de l'arrimage local de modèles globalisés de ville nouvelle qu'articulent l'action publique urbaine chinoise. Cette seconde mondialisation est très ramassée dans le temps; ces trois inflexions se succèdent et cohabitent à la fois.

\section{Conclusion : Vers une trajectoire de la ville nouvelle chinoise de desserrement}


Les événements politiques et économiques qui se sont succédés depuis la fondation de la République populaire de Chine ont engagé les villes chinoises dans une trajectoire d'industrialisation et d'urbanisation singulières (Ma, 2002). Dès lors, une transition urbaine (Lewis, 1954) proprement chinoise se dessine (Friedmann, 2006), segmentée en plusieurs phases; chacune correspond à des processus de territorialisation différenciés et à la mise en place de modèles de villes nouvelles variés (Henriot, 2013b : 63-90 ; Henriot, 2015).

Tableau 2 : La trajectoire chinoise des modèles de ville nouvelle et sa mondialisation (réalisé par l'auteur)

\begin{tabular}{|c|c|c|c|}
\hline $\begin{array}{l}\text { Contexte } \\
\text { historique }\end{array}$ & Processus de territorialisation & Type de ville nouvelle & Influence \\
\hline $\begin{array}{l}\text { 1949-1977 } \\
\text { Les trente } \\
\text { premières années } \\
\text { du régime } \\
\text { communiste }\end{array}$ & $\begin{array}{l}\text { - Urbanisation retardée } \\
\text { - Industralisation avec } \\
\text { extension urbaine limitée } \\
\text { - Desserrement industriel } \\
\text { - « under-urbanization » } \\
\text { (Kirkby, 1987) } \\
\text { - « intense industrialization } \\
\text { with a controlled } \\
\text { urbanisation », " latent } \\
\text { urbanisation » } \\
\text { (Ma, 2002: 1550) } \\
\text { - « incomplete urbanization » } \\
\text { (Chan, 2010) } \\
\text { - " suburbs as state invested } \\
\text { industrial space » } \\
\text { (Wu et Shen, 2015) }\end{array}$ & $\begin{array}{l}\text { - Cité ouvrière, } \\
\text { "village " (xun) ou } \\
\text { "nouveau village " } \\
\text { (xincun), à l'échelle } \\
\text { micro } \\
\text { Ex : Caoyang xincun } \\
\text { (Shanghai), Nanyan } \\
\text { fang, Shenghuo cun } \\
\text { (Wuhan) } \\
\text { - Ville satellites } \\
\text { (weixincheng), à } \\
\text { l'échelle macro } \\
\text { Ex : Minhang, } \\
\text { Baoshanwei, } \\
\text { Jinshanwei (Shanghai) - } \\
\text { Shijingshan, Yanshan } \\
\text { (Pékin) }\end{array}$ & $\begin{array}{l}\text { - Soviétique } \\
\text { - Influence de la } \\
\text { ville nouvelle } \\
\text { soviétique } \\
\text { - Aide à la } \\
\text { planification } \\
\text { d'experts } \\
\text { soviétiques } \\
\text { jusqu'en } 1960\end{array}$ \\
\hline $\begin{array}{l}\text { 1978-1991 } \\
\text { Les réformes et } \\
\text { l'ouverture }\end{array}$ & $\begin{array}{l}\text { - Urbanisation latente } \\
\text { - Industrialisation des } \\
\text { campagnes littorales } \\
\text { - « bottom-up urbanization », } \\
\text { « de facto urbanization » (Ma, } \\
\text { 2005) } \\
\text { - « Industrialisation des } \\
\text { campagnes » (Sanjuan, 2000) } \\
\text { - « industrial } \\
\text { decentralization » (Wu et } \\
\text { Shen, 2015) } \\
\text { - « rural industrialisation, } \\
\text { scattered development and } \\
\text { the forming of city regions } \\
\text { (1980s) (Yeh et Chen, 2020) }\end{array}$ & $\begin{array}{l}\text { Renouveau de la ville } \\
\text { satellite }\end{array}$ & $\begin{array}{l}\text { - Echanges } \\
\text { internationaux } \\
\text { limités }\end{array}$ \\
\hline $\begin{array}{l}\text { 1992-1999 } \\
\text { Le « renouveau } \\
\text { urbain » } \\
\text { (Sanjuan 2000) }\end{array}$ & $\begin{array}{l}\text { - Urbanisation récente et } \\
\text { accélérée } \\
\text { - « Différenciation et } \\
\text { spécialisation de l'espace } \\
\text { urbain » (Larivière, 1997) } \\
\text { - « Land-centered growth, fast } \\
\text { urban expansion and }\end{array}$ & $\begin{array}{l}\text { - Ville nouvelle } \\
\text { (xincheng) } \\
\text { Ex : Shijingshan, } \\
\text { Yanshan (Pékin) }\end{array}$ & $\begin{array}{l}\text { - Internationale } \\
\text {-Planification } \\
\text { traditionnelle } \\
\text { - Fonctionnalisme } \\
\text { - Zonage } \\
\text { - Desserrement } \\
\text { polycentrique }\end{array}$ \\
\hline
\end{tabular}




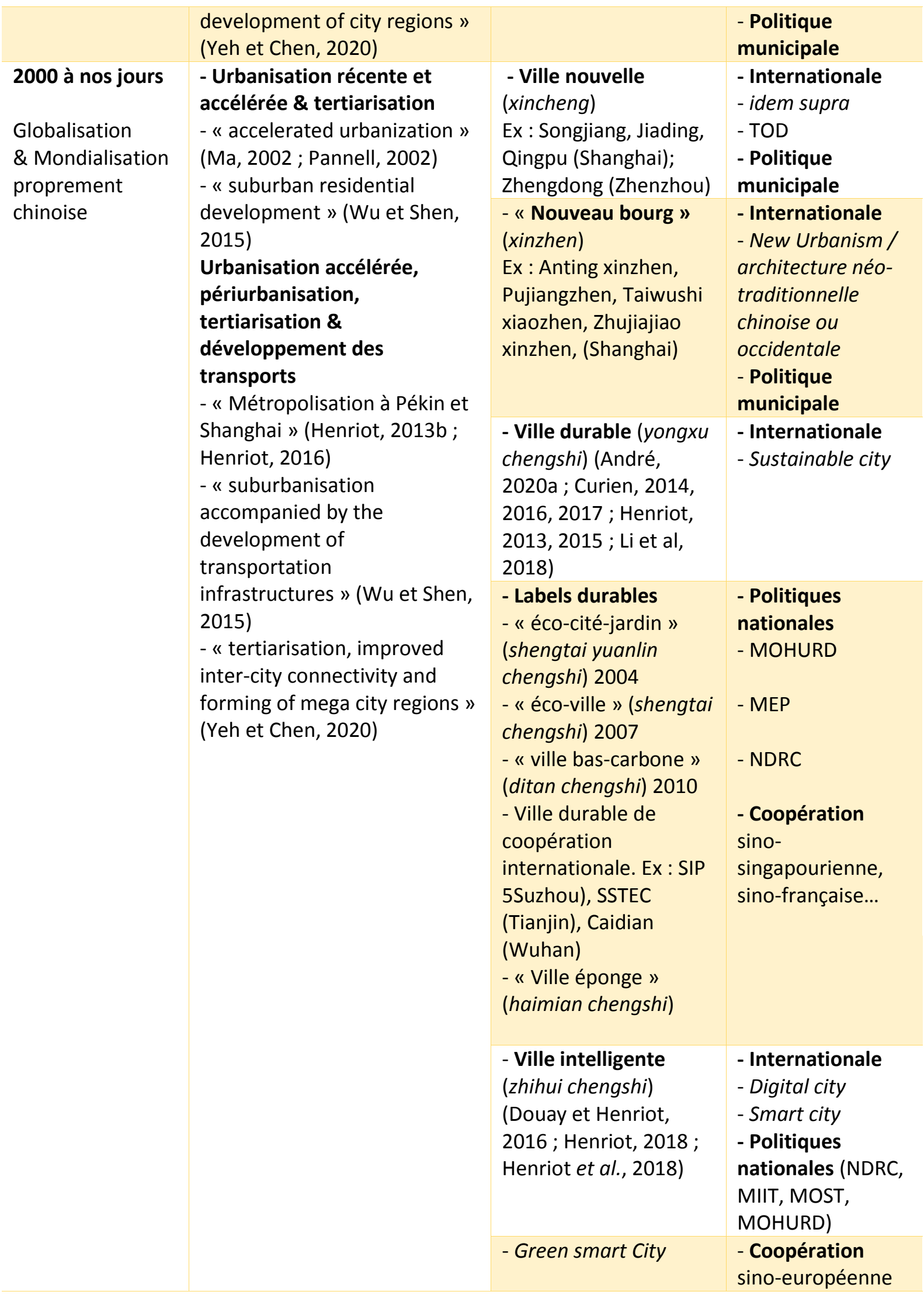

La ville nouvelle chinoise dessine une trajectoire qui lui est propre, pour s'inscrire au sein de la mondialisation de la ville nouvelle, de la circulation de ses modèles d'urbanisme et de ses références urbaines. Cette trajectoire proprement chinoise se caractérise d'abord par des emprunts à la 
planification soviétique, ce qui est inédit en Asie orientale, où les expériences japonaise, coréenne et hongkongaise bénéficient plutôt de l'expertise des villes nouvelles britanniques et secondairement françaises. Les premières villes nouvelles chinoises de décongestion élaborées dans un contexte d'industrialisation soutenue se multiplient, entre le début des années 1960, jusqu'à la fin des années 1980, sous la forme de villes satellites industrielles et d'habitat ouvrier. Progressivement, à partir des années 1990, sous l'intervention d'experts internationaux (coopération technique bilatérale ou multilatérale, bailleurs de fonds, agences d'architecture-urbanisme, bureaux d'études techniques), la ville nouvelle chinoise de desserrement s'inscrit dans la planification traditionnelle, le fonctionnalisme et le zonage. Au début des années 2000, l'intégration croissante de la République populaire de Chine à la globalisation, renouvelle les approches de la ville nouvelle chinoise, laquelle s'arrime désormais à une seconde mondialisation, infléchie par le nouvel urbanisme et l'architecture néo-traditionnelle, la ville durable, mais également les approches numériques et pro-technologiques de l'urbanisme. Ainsi les villes nouvelles contemporaines chinoises de desserrement urbain constituent-elles un observatoire des objectifs d'industrialisation, puis d'urbanisation, répondant à des besoins quantitatifs - bien qu'un affiche qualitatif récent soit revendiqué. Les projets chinois ne présentent absolument pas les ambitions théoriques des villes nouvelles britanniques du New Town Act. Les villes nouvelles chinoises restent des expérimentations très pragmatiques et partagent, avec l'expérience française, une même référence au "laboratoire " et au projet urbain - lequel permet " une convergence des modèles et des intérêts élaborés à des échelles et par des acteurs différents " (André, 2020a : 189).

Loin d'appartenir à l'histoire de l'urbanisme, la ville nouvelle chinoise constitue un outil privilégié articulant planification urbaine et projet urbain, inscrit dans la mondialisation de la ville nouvelle, adapté localement et renouvelé selon les inflexions de l'action publique locale et nationale. Les villes nouvelles de desserrement chinoises se signalent " par l'arrimage des ambitions locales dans les modèles nationaux et transnationaux " (André, 2020a : 199). La ville nouvelle chinoise est ainsi à la fois "a backup space" (Sampieri, 2019) et "a container of worldwide recycled models" (Fiandanese, 2019). Au-delà, chaque expérimentation chinoise participe d'une mondialisation asiatique de certaines formes urbaines ( $C B D$, condominium, shopping mall) et de certains outils de l'aménagement contemporain, comme la zone franche économique - et la ville nouvelle, véritable laboratoire de la production urbaine (extra)ordinaire.

\section{Bibliographie :}

André, G., 2016. "La métropolisation à marche forcée: les effets de la coordination entre les politiques de transport et d'urbanisme à Wuhan ". L'Information géographique, vol. 80, n 3, p 26-39. https://doi.org/10.3917/lig.803.0026

André, G., 2020a. «Temporalités de la métropolisation et appropriations locales : les mutations des secteurs résidentiels à Wuhan, une grande ville chinoise de l'intérieur ", thèse de doctorat en géographie, université Paris 1 Panthéon-Sorbonne, $667 \mathrm{p}$

André, G., 2020b. "Wuhan, d'un centre industriel secondaire à une "Chicago de I'Est" ", Géoconfluences, URL : http://geoconfluences.ens-lyon.fr/informations-scientifiques/dossiersregionaux/la-chine/articles-scientifiques/wuhan

Antier, G. et G. Abadia, 1987. « La longue marche de Shanghai ». Cahiers de I'IAURIF, n 81, p. 49-58.

Antier, G., 1989. «Villes nouvelles, extérieur nuage ». Cahiers de l'IAURIF, n 87-88, p. 139-145. 
Baeumler, A., E. Ijjasz-Vasquez, S. Mehndiratta, 2012. Sustainable Low-Carbon City Development in China. World Bank Publications, 588 p.

http://siteresources.worldbank.org/EXTNEWSCHINESE/Resources/3196537-

1202098669693/4635541-1335945747603/low carbon city full en.pdf

Ballout, J.M., 2019, "Devenirs de(s) villes nouvelles dans les Suds », Appel à contribution, Calenda, Publié le mercredi 03 avril 2019, https://calenda.org/594821.

Ballout, J.M., 2020, « Edito : direction du dossier thématique », COM.

Banque asiatique de développement, 2010. "Sustainable Urban Development in the People's Republic of China. Eco-City Development - a new and sustainable way forward? ". Urban Innovations and best Practices, $8 \mathrm{p}$.

Banque mondiale, 2009. Sino-Singapore Tianjin Eco-City: A case study of an Emerging Eco-City in China. Technical Assistance Report, World Bank Publications, 142 p.

Bergère, M.-C., 2002. Histoire de Shanghai. Paris, Fayard, 520 p.

Billard, G., A. Cordier, L. Lemoine, 2005. "Riverside, anatomie d'une ville nouvelle chinoise ». Les Annales de la Recherche Urbaine, $n^{\circ}$ 98, Paris, Centre de recherche et d'urbanisme, p. 159-166.

Bonino, M., F. Governa, M.-P. Repellino, A. Sampieri (dir.), 2019. The City after Chinese New Towns, Spaces and Imaginaries from Contemporary Urban China. Birkhauser, Basel, 253 p.

Cagnardi, A., 2003. « Un'avventura affascinante : Pujiang ». Urbanistica, n 122, p. 63-74.

Cao, S, 1994. "Shanghai 1292-1992: processus de formation de la ville dans ses relations aux facteurs économiques et politiques ». Thèse de doctorat en géographie, Université Paris 1 PanthéonSorbonne, $320 \mathrm{p}$.

Chaline, C., 1985, réed. 1996. Les villes nouvelles dans le monde. Paris, PUF, n²231, 127 p.

Chan, K.-W., 2010. "Fundamentals of China's urbanization and policy », The China Review, vol. 10, $\mathrm{n}^{\circ}$ 1, p. 79.

URL: http://faculty.washington.edu/kwchan/Chan-Fundamentals-CR2010.pdf, consulté le 1/05/2015.

Choay, F., 1988, réed. 2015. " modèle ", in P. Merlin, F. Choay (dir.), Dictionnaire de l'urbanisme et de l'aménagement, Paris, PUF, p. 472.

Chow, C.K., 1991. "New towns development in Hong Kong ", in P. Merlin et M. Sudarskis, 1991, "From garden city to urban reconstruction: new towns in perspective", actes du colloque de Etgham, Den Haag, INTA/AIVN Press, avril 1991, pp. 45-165.

Curien, R., 2014. « La planification des villes chinoises ». Perspectives chinoises, n³, p. 27-35.

Curien, R., 2016. « L'éco-cité de Tianjin : innovations et limites d'une conception sino-singapourienne d'une ville durable ", Métropolitiques. URL : https://metropolitiques.eu/L-eco-cite-de-Tianjininnovations.html

Curien, R., 2017. "Singapour, modèle de développement urbain (durable ?) en Chine ", Perspectives chinoises, 2017/1, p. 27-37. 
Dell, J., A. Speer, 2002. " Anting New Town, Shanghai. Europäische Planungsprinzipien in Asiens Boomtown ». Trialog 75, Planen und Bauen in der Dritten Welt, $n^{\circ} 4$, Dossier New Settlements, p. 1720.

Delpirou, A., J.-F. Doulet, J. Zhuo, 2015. "Coordonner urbanisme et transports collectifs : un référentiel à l'épreuve de la ville "made in China" ». Flux, n¹01-102, p. 42-56.

Den Hartog, H., 2010 (dir.). Shanghai new towns : searching for community and identity in a sprawling metropolis, Rotterdam, 010 Publishers, $416 \mathrm{p}$.

De Pieri F., D. Vero, 2019. « When ends dont' meet. Historical interpretations of twenty-first century new towns ", in M. Bonino, F. Governa, M.-P. Repellino, A. Sampieri (dir.), The City after Chinese New Towns, Spaces and Imaginaries from Contemporary Urban China, Birkhauser, Basel, p. 71-77.

Doulet, J.F., 2015. "L'urbanisme chinois et l'émergence du modèle "intégrationniste" ». Métropolitiques. URL : http://www.metropolitiques.eu/L-urbanisme-chinois-et-I-emergence.html

Etteinger, B., 1992. "Yi Zhuang : un projet de ville nouvelle dans la région de Beijing ", Rapport de mission du 15-28/05/1992, réalisé pour l'IAURIF, 41 p.

EU-China Smart and Green City Cooperation, 2014. "Comparative Study of Smart Cities in Europe and China ", Current Chinese Economic Report Series, Springer, 261 p.

Fiandanese, F., 2019. « Transational Models. A study on Tongzhou New Town ». Doctoral Dissertation in Architecture, History and Project, Politecnico di Torino, 229 p.

Fouchier, V., 1993. " Hong Kong : Comment gérer les fortes densités ? ». Perspectives chinoises, $\mathrm{n}^{\circ} 13-$ 14 , p. 56-66.

Friedmann, J., 2006. "Four theses in the study of China's urbanization ». International Journal of Urban and Regional Research, vol. 30, n², p. 440-451.

Fung, K.-I., 1981a. "The spatial development of Shanghai ", in C. Howe, Shanghai. Revolution and development in an Asian metropolis, Cambridge, Cambridge University Press, p. 269-300.

Fung, K.-I., 1981b. "Satellite town development in the Shanghai city region ». Town Planning review, vol. $52, n^{\circ} 1$, p. 26-46.

Funk, K.-I., 1996. "Satellite towns: development and contributions ", in Y.-M. Yeung, Y.-W. Sung (dir.), Transformation and modernization under China's Open Policy, Hong Kong, The Chinese University Press, p. 321-340.

Ged, F., 2000. Shanghai. Paris, Institut français d'architecture, 64 p.

Gélézeau, V., 2003. Séoul, ville géante, cités radieuses. Paris, CNRS éditions, p. 72

Gélézeau, V., 2011. Séoul. Paris, Autrement, p. 58-59.

Henriot, C., 2013a. "Aménager les périphéries urbaines chinoises : ville nouvelle et partenariat public-privé à Shanghai ». URBIA. Les cahiers du développement urbain durable, hors-série $n^{\circ} 1$ 
"Urbanisme et aménagement du territoire, un aperçu de la jeune recherche francophone ", p. 207222.

Henriot, C., 2013b. "Villes nouvelles et redéploiement métropolitain à Shanghai. Les nouvelles périphéries urbaines chinoises ", thèse de doctorat en géographie, université Paris 1 PanthéonSorbonne, $440 \mathrm{p}$.

Henriot, C., 2014. « Les logements sociaux du « Nouveau Triomphe » à Shanghai : facteurs de fragmentation sociale et spatiale ", dans F. Belmessous, L. Bonneval, L. Coudroy De Lille, N. Ortar, 2014. Logement et politique(s). Un couple encore d'actualité ?, Paris, L'Harmattan, p. 85-102.

Henriot, C., 2015. « Les politiques chinoises de villes nouvelles : trajectoire et ajustements de l'action publique urbaine à Shanghai ». Géocarrefour, 90/1, p. 27-38. ISSN électronique 1960-601X, DOI : 10.4000/geocarrefour.9659.

Henriot, C., 2016. "Métropolisation chinoise et villes nouvelles : l'exemple de l'aménagement polycentrique de Shanghai ». Géoconfluences, mis en ligne le 14 février 2016 URL : http://geoconfluences.ens-lyon.fr/informations-scientifiques/dossiers-regionaux/lachine/corpus-documentaire/villes-nouvelles

Henriot, C., 2017a. "À l'ombre de Shanghai : une petite ville nouvelle, relais de l'urbanisation et de l'urbanité métropolitaine » \& [English version] "In the shadow of Shanghai: A new small town, relay of urbanisation and metropolitan urbanity ». L'Espace géographique, 2017-4, p. 329-345.

Henriot, C., 2017b. "Peupler les villes nouvelles shanghaïennes et devenir urbain », in C. Mazzoni, L. Fan, A. Grigorovschi, Y. Liu (dir.), SHANGHAl, Kaleidoscopic city, Paris, Éditions La Commune, p. 125135.

Henriot, C., 2018. " La politique de la ville intelligente en Chine : ancrage local d'un modèle urbain globalisé ». Flux, n¹14, p.71-85. DOI : 10.3917/flux1.114.0071

Henriot, C., N. Douay, B. Granier, R. Languillon-Aussel, N. Leprêtre, 2018. « Perspectives asiatiques sur les Smart Cities . Flux, n¹14, p.1-8. DOI : 10.3917/flux1.114.0001

Henriot, C., M. Minost, 2017. "Thames Town, un cliché à l'anglaise. Production urbaine et construction sociale d'un quartier d'architecture occidentale à Shanghai ». Perspectives chinoises, 2017/1, p. 83-90. ISSN électronique 1996-4609 \& [English version] « Thames Town, an English Cliché: The Urban Production and Social Construction of a District Featuring Western-style Architecture in Shanghai ». China Perspectives, 2017/1, p. 79-86. Electronic ISSN 1996-4617.

Henry, M., 1984. "Pékin, Shanghai et la région Île-de-France, un début d'échange d'informations ». Cahiers de I'IAURIF, n 73, p. 65-70.

Hoa, L., 1981. Reconstruire la Chine, trente ans d'urbanisme, 1949-1979. Paris, Le Moniteur, p. 63-65.

Keeton, R. (dir.), 2011. Rising in the East: contemporary new towns in Asia. SUN Architecture, Amsterdam. 
Kirkby, R.J.R., 1987. "A review of satellite town policies in the People's Republic of China. The experience of Shanghai ", in D. Philips et A. Yeh (dir.), New towns in East and South East Asia, Hong Kong, Oxford University Press, p. 205-230.

Larivière, J.P., 1997. "Pékin : métamorphose d'une capitale ", L'Information Géographique, vol.61, $\mathrm{n}^{\circ} 2, \mathrm{p} .55-64$.

Lee, T.-I. 1987. "New town planning and development in Korea », in D. Philips et A. Yeh (dir.), New towns in East and South East Asia, Hong Kong, Oxford University Press, p. 109-125.

Lewis, A., 1954. « Economic Development with Unlimited Supply of Labour ». The Manchester School of Economic and Social Studies, vol. 47, n³, p. 139-191.

Li, J., R. Chiu, 2018. "Urban investment and development corporations, new town development the case of Songjiang new town, Shanghai ». Urban geography, vol. 39, p. 687-705.

Li, X., 2010. « Heterotopias: Themed Spaces in Shanghai and Los Angeles », in H. Den Hartog (dir.), 2010, Shanghai New Towns: Searching for Community and Identity in a Sprawling Metropolis, Rotterdam, 010 Publishers, p. 223-238.

$\mathrm{Li}, \mathrm{X}, 1991$. "Problèmes posés par les villes satellites de Shanghai ". Mémoire de DEA en aménagement et urbanisme, Institut d'urbanisme de Paris, Université Paris XII Val-de-Marne, Créteil, $47 \mathrm{p}$.

Li, Y., C. Bonhomme, J.-F. Deroubaix «Un modèle de développement urbain durable peut-il s'exporter ? La construction de "l'exemplaire " éco-cité de Tianjin ", Perspectives chinoises, 2018/12, p. 93-104.

Li, Z., X. Li, Wang L., 2014. « Speculative urbanism and the making of university towns in China: A case of Guangzhou University Town », Habitat International, vol. 44, p. 422-431.

http://dx.doi.org/10.1016/j.habitatint.2014.08.005

Lin, G., D. Wei, 2002. "China's restless urban landscapes: new challenges for theoretical reconstruction ». Environment and Planning A, vol. 34, n 9, p. 1535-1536.

Liu, J., G. Xu, 2019. "A policy discourse on new town development in contemporary china ", in M. Bonino, F. Governa, M.-P. Repellino, A. Sampieri (dir.), The City after Chinese New Towns, Spaces and Imaginaries from Contemporary Urban China, Birkhauser, Basel, p.60-70.

Liu, Y., 2011. " Shanghai : villes nouvelles et inspirations étrangères ", in C.-N. Douady (dir.), EuroOrient, n³3-34, "Espaces chinois urbains et culturels », p. 101-120.

Liu, Y., 2014. "Les villes nouvelles de Shanghai : rôle et fonctions dans la structuration de la métropole et mixité fonctionnelle à Jinshan ", thèse de doctorat en architecture. Paris : Université Paris-Est, 485 p.

Liu, Y., 2018. Jinshan, une ville nouvelle dans la métropole de Shanghai. Paris, PUF, Presses universitaires de Rennes, $213 \mathrm{p}$.

Lorrain, D., 2010. " "Portrait d'entreprise » Blue red chips : les compagnies d'eau en Chine ». Flux, $\mathrm{n}^{\circ} 82$, p. 64-80. 
Lorrain, D., 2011. "Shanghai ou une modernisation publique ", in D. Lorrain (dir.), Métropoles XXL en pays émergents, Paris, Les Presses de Science Po, p. 53-138.

Lu, D., Y. Tian, V.Y. Liu, Y. Zhang, 2015. " The performance of the smart cities in China -a comparative study by means of self-organizing maps and social networks analysis ». Sustainability, 7, p.7604-7621.

Ma, L.J.C., 2002. "Urban transformation in China, 1949-2000: a review and research agenda ». Environment and planning A, vol. 34, $n^{\circ}$ 9, p. 1545-1569.

Ma, L.J.C., 2005. " Privatization of rural industry and de facto urbanization from below in Southern Jiangsu, China ». Geoforum, n 36, pp. 761-777.

McCann, E., K. Ward (dir.), 2011. Mobile Urbanism: City Policymaking in the Global Age. Minneapolis, University of Minnesota Press.

McCann, E., K. Ward, 2012. "Policy assemblages, mobilities and mutations: Toward a multidisciplinary conversation ». Political Studies Review, vol. 10, n³, p. 325-332.

Merlin, P., 1969, rééd. 1972. "L'urbanisme en région de Londres et les villes nouvelles ", in P. Merlin, Les villes nouvelles. Urbanisme régional et aménagement, Paris, Presses universitaires de France, p. 11-82.

Merlin, P., 1988, rééd. 1996, "Ville nouvelle ", in P. Merlin, F. Choay, 1996, Dictionnaire de l'urbanisme et de l'aménagement, Paris, PUF, p. 847-850.

Merlin, P., M. Sudarskis, 1991. "From garden city to urban reconstruction: new towns in perspective ", actes du colloque de Etgham, Den Haag, INTA/AIVN Press, avril 1991, p. 45-165.

Murphey, R., 1976. "Chinese urbanization under Mao ", in B.J.L. Berry (dir.), Urbanization and Counter-urbanization, Urban Affairs Annual Reviews, vol. 11, CA, Beverley Hills, Sage, p. 310-329.

Nagamine, H., 1987. « New town development in Japan : an experience of muddling throught », in D. Philips, A. Yeh (dir.), New Towns in East and South-east Asia, Hong Kong, Oxford University Press, p. 170-204.

Nieuwenhuis, M., 2010. " Tracing the Politics of Space in One City \& Nine Towns ", in H. Den Hartog (dir.), Shanghai New Towns: Searching for Community and Identity in a Sprawling Metropolis, Rotterdam, 010 Publishers, p. 291-304.

Ning, Y., Yan Z., 1995. "The changing industrial and spatial structure in Shanghai ». Urban Geography, vol. 16, n 7, p. 577-594.

Orillard, C., 2017. Acteurs de la " politique française des villes nouvelles » et études à l'export: Le cas de l'action en Argentine. Histoire urbaine, vol. 50, n³, p. 143-160. doi:10.3917/rhu.050.0143.

Pannell, C., 2002, "China's continuing urban transition ». Environment and Planning A, vol. 34, $n^{\circ}$ 9, p. 1571-1589.

Piazzoni M. F., T. Banerjee, 2018. " Mimicry in design: the urban form of development ", Journal of Urban Design, vol. 23, n4, p. 482-498. Doi: 10.1080/13574809.2017.1383151 
Petit, O., 2002. "L'influence des villes nouvelles françaises en Asie, dans leur rapport avec les idées, les entreprises et les hommes de l'art français ", Programme interministériel d'histoire et d'évaluation des villes nouvelles françaises, La Défense, ISTED « Villes en développement », 115 p.

Philips, D., Yeh A. (dir.), 1987. New Towns in East and South East Asia. Hong Kong, Oxford University Press, $\mathrm{p}$.

Pun, K.S. 1987. "New towns and urban renewal in Hong Kong ", in D. Philips, A. Yeh (dir.), New Towns in East and South-east Asia, Hong Kong, Oxford University Press, p. 41-58.

Ruoppilaa S., F. Zhao, 2017. The role of universities in developing China's university towns: The case of Songjiang university town in Shanghai. Cities, vol. 69, p. 56-63.

Sampieri, A., 2019. "The city is available. Chinese new towns as a backup space ", in M. Bonino, F. Governa, M.-P. Repellino, A. Sampieri (dir.), The City after Chinese New Towns, Spaces and Imaginaries from Contemporary Urban China, Birkhauser, Basel, 253p. p. 204-214.

Sanjuan, T., 2000. La Chine. Territoire et société. Paris, Hachette, 189 p.

Sanjuan, T., 2004. La Chine en recomposition : pouvoirs territoriaux, dynamiques urbaines et globalisation. Habilitation à diriger des recherches en géographie, Université Paris 1 PanthéonSorbonne, 3 tomes : 75, 229, $233 \mathrm{p}$.

Shen, J., F. Wu, 2012. "Restless urban landscapes in China: a case study of three projects in Shanghai ». Journal of Urban Affairs, vol. 34, n 3, p. 236.

Shen, J., F. Wu, 2017. « The Suburb as a Space of Capital Accumulation: The Development of New Towns in Shanghai, China ». Antipode, vol. 49, n³, p. 761-780. Doi: 10.1111/anti.12302

Shen, J., F. Wu, 2019. "Paving the way to growth: transit-oriented development as a financing instrument for Shanghai's post-suburbanization ". Urban Geography, p. 1-23. Doi: $\underline{10.1080 / 02723638.2019 .1630209}$

Tan, X., 2010, "New-Town Policy and Development in China ". The Chinese Economy, vol. 43, n³, p. 47-58.

Thompson, R., 1974. « Containing the City ». Architectural Design, p. 152.

Vadelorge, L., (dir.), 2005. Gouverner les villes nouvelles. Le rôle de l'Etat et des collectivités locales. Le Manuscrit, $405 \mathrm{p}$.

Vadelorge, L., (dir.), 2014. Retour sur les villes nouvelles. Une histoire urbaine du XXe siècle. Paris, Créaphis, $464 \mathrm{p}$.

Vadelorge, L., (dir.), 2017a. "Circulations internationales de modèles et d'expériences des villes nouvelles ». Histoire urbaine, vol. 50, $\mathrm{n}^{\circ} 3$.

Vadelorge, L., 2017b, " Introduction ", Histoire urbaine. vol. 50, n³, p. 67-72, doi:10.3917/rhu.050.0067. 
Vadelorge, L. 2017c. "Le tropisme international des villes nouvelles françaises dans les années 1970: Pratiques, enjeux et contextes de trois voyages d'études: Québec, Arabie Saoudite, Chine ». Histoire urbaine, vol. 50, n³, p. 161-178. doi:10.3917/rhu.050.0161.

Wang, Z. et Li Z., 2006. " "Yi cheng jiu zhen", duijiaoqu xinchengzhen de qishi », [Éclaircissements sur les bourgs nouveaux de banlieue du programme "une ville, neuf bourgs"]. Jianzhu xuebao [Architectural Journal], $n^{\circ}$ 7, p. 8-11.

Wang, Z., 2007. " Shanghai "yi cheng jiu zhen" kongjian jiegou ji xingtai leixing yanjiu » [Morphologie et structure spatiale des " une ville, neuf bourgs » de Shanghai]. Thèse de doctorat en architecture et en urbanisme, Université de Tongji, Shanghai, 272 p.

Wu, Y., W. Zhang, J. Shen, Z. Mo et Y. Peng, 2018. " Smart City with Chinese caracteristics against the background of big data : idea, action and risk ». Journal of Cleaner Production, $n^{\circ} 173$, p. 60-66.

Yeh, A., 1987. "Spatial Impacts of New Town Development in Hong Kong », in D. Philips, A. Yeh (dir.), New Towns in East and South-East Asia, Hong Kong, Oxford University Press, p. 59-81.

Yeh, A. G.-O., Z., Chen, 2020. "From cities to super mega city regions in China in a new wave of urbanisation and economic transition: Issues and challenges ». Urban Studies, vol. 57, n³, p. 636654.

Yu, S., Z. Luo, 2009. "Shanghai jiaoqu xincheng de guihua yu sikao " [Planification et réflexions sur les villes nouvelles de la banlieue de Shanghai]. Chengshi guihua xuekan [Cahiers d'urbanisme], vol. $3, n^{\circ} 181$, p. 13-19.

Zhang, S. et al., 2015. «PPP application in infrastructure development in China: Institutional analysis and implications ». International Journal of Project Management, vol. 33, p. 497-509.

Zhang, Y. et al., 2014. "From State to Market: Private Participation in China's Urban Infrastructure Sectors, 1992-2008 ». Word Development, vol. 64, p. 473-486.

Zhou, M., Q., Xue, 2008. " "Otherness" strategy: the Origin of "One City, Nine Towns" Plan in Shanghai». Guoji chengshi guihua [International Urban Planning], 2008, Vol. 23, n² 2, p.113-117.

Zhou, N., G. He, C. Williams, 2012. "China's Development of Low-Carbon Eco-Cities and Associated Indicator Systems ", Rapport de recherche rédigé pour China Energy Group, Energy Analysis \& Environmental Impacts Department, Environmental Energy Technologies Division, Ernest Orlando Lawrence, Berkeley National Laboratory.

URL: http://china.lbl.gov/sites/all/files/china eco-cities indicator systems.pdf

Zhuang, L., C. Ye, 2018. « Disorder or Reorder? The Spatial Production of State-Level New Areas in China ». Sustainability, vol. 10, n¹0, p. 3628. doi:10.3390/su10103628

Zhuo, J., 2004. "Mobilité urbaine en Chine : enjeux et problématiques. Le cas de Shanghai et d'autres grandes métropoles ", conférence MUTA, Mont Tremblant, N.R.

Zhuo, J., 2015. "TOD, un paradigme urbanistique à l'épreuve de la ville chinoise: le cas de Shanghai ", Rencontres internationales de l'APERAU, Rennes, 1-5/06/2015. 